\title{
Spatially explicit models of dynamic histories: examination of the genetic consequences of Pleistocene glaciation and recent climate change on the American Pika
}

\author{
JASON L. BROWN* and L. LACEY KNOWLES+ \\ *Department of Biology, Duke University, 125 Science Drive, Durham, NC 27705, USA, +Department of Ecology and \\ Evolutionary Biology, Museum of Zoology, University of Michigan, Ann Arbor, MI 48109-1079, USA
}

\begin{abstract}
A central goal of phylogeography is to identify and characterize the processes underlying divergence. One of the biggest impediments currently faced is how to capture the spatiotemporal dynamic under which a species evolved. Here, we described an approach that couples species distribution models (SDMs), demographic and genetic models in a spatiotemporally explicit manner. Analyses of American Pika (Ochotona princeps) from the sky islands of the central Rocky Mountains of North America are used to provide insights into key questions about integrative approaches in landscape genetics, population genetics and phylogeography. This includes (i) general issues surrounding the conversion of time-specific SDMs into simple continuous, dynamic landscapes from past to current, (ii) the utility of SDMs to inform demographic models with deme-specific carrying capacities and migration potentials as well as (iii) the contribution of the temporal dynamic of colonization history in shaping genetic patterns of contemporary populations. Our results support that the inclusion of a spatiotemporal dynamic is an important factor when studying the impact of distributional shifts on patterns of genetic data. Our results also demonstrate the utility of SDMs to generate species-specific predictions about patterns of genetic variation that account for varying degrees of habitat specialization and life history characteristics of taxa. Nevertheless, the results highlight some key issues when converting SDMs for use in demographic models. Because the transformations have direct effects on the genetic consequence of population expansion by prescribing how habitat heterogeneity and spatiotemporal variation is related to the species-specific demographic model, it is important to consider alternative transformations when studying the genetic consequences of distributional shifts.
\end{abstract}

Keywords: climate change history, coalescent simulations, demographic models, phylogeography, spatiotemporal dynamic

Received 15 November 2011; revision received 22 March 2012; accepted 2 April 2012

\section{Introduction}

Species are inherently connected to the environment in which they evolve. For many species, this represents a constant changing of the regional mosaic of habitats. A lack of stability in climate and habitat throughout the

Correspondence: Jason L. Brown, Fax: (919) 660-7293;

E-mail: jasonleebrown@gmail.com history of a species has many genetic consequences (Hewitt 1996; Knowles \& Richards 2005; Lohse et al. 2011). In many cases, distributional shifts of species have been dramatic (e.g. owing to Pleistocene glaciation or sea level changes), and locations of past populations do not correspond to contemporary distributions (e.g. Carstens \& Richards 2007; Knowles et al. 2007; Galbreath et al. 2009; Voje et al. 2009; Graham et al. 2010; Morgan et al. 2011). Species respond to changes in 
climate, and resulting habitat changes, by migrating to new suitable areas (habitat tracking), adapting to changes in situ, adjusting to changes by means of phenotypic plasticity, dying out, or some combination of these. A central goal of phylogeography is to identify and characterize the processes underlying divergence. One of the biggest impediments currently faced is how to capture the spatiotemporal dynamic under which a species evolved (Fig. 1). The integration of statistical phylogeography and geospatial methods has begun to elucidate approaches for assessing this and allows researchers to derive hypothesis from one approach that can be tested (e.g. placing demographic events in a historical and spatial context; e.g. see Knowles 2009; Chan et al. 2011; Manolopoulou et al. 2011).

A recent proposal to couple species distribution models (SDMs), demographic and genetic models illustrated how they can be used to generate species-specific expectations for patterns of genetic variation (Knowles \& Alvarado-Serrano 2010). Knowles \& Alvarado-Serrano (2010) demonstrated that movement through a heterogeneous environment has genetic consequences, as do species-specific life histories (see also Excoffier et al. 2009 for a review of theory on spatial expansion). Consequently, we expect species to have different genetic signatures of past population movements, despite migrating through the same region. Here, we focus specifically on the challenges of the proposal (Knowles \& Alvarado-Serrano 2010) to expand statistical phylogeographic frameworks that integrate SDMs and genetic models (e.g. Richards et al. 2007; Carnaval et al. 2009; Knowles 2009; Chan et al. 2011) to include a demographic model that can capture differences in speciesspecific life histories and degrees of ecological specialization. Our focus here is on translating SDMs into demographic models (i.e. the conversion of habitat suitability scores into population demographic parameters, such as carrying capacities). We also focus on how to connect past and present SDMs, and in doing so, we examine specifically how the temporal dynamic of distributional shifts over time impact patterns of genetic variation in species. With the obvious complications with obtaining detailed information on the spatiotemporal dynamic of climate-induced distributional shifts, we compare differ-

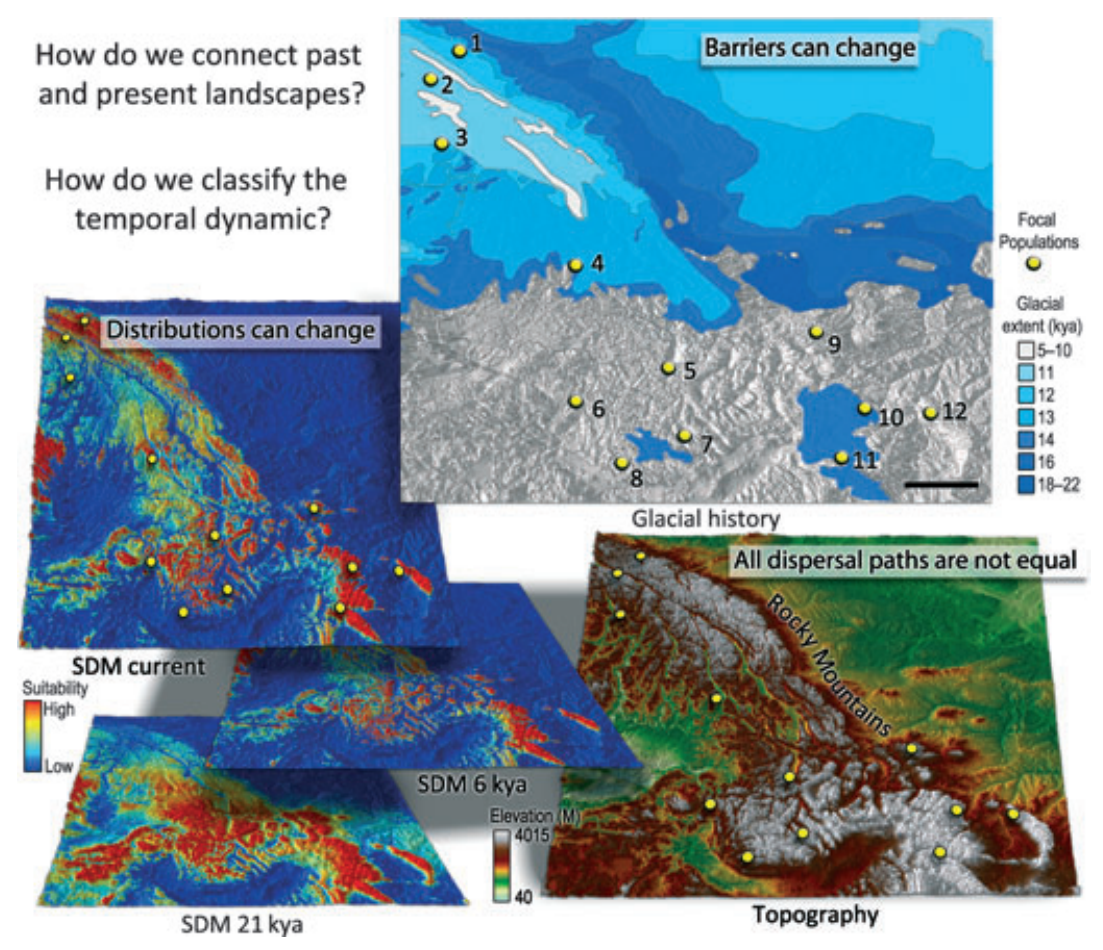

Fig. 1. The main research questions illustrated by geospatial data incorporated into this study (clockwise from top). Glacial distribution since the last glacial maximum. Yellow dots depict focal populations of the study: 1. Grande Cache, AB; 2. McBride, BC; 3 . Raft Mtn., BC; 4. Roman Nose Lakes, ID; 5. Darby, MT; 6. Black Lake, ID; 7. Doublespring Pass, ID; 8. Featherville, ID; 9. Neihart, MT; 10. Red Lodge, MT; 11. Togwotee Pass, WY; 12. Duncum Mtn., WY. Northern populations (1-3) colonized this habitat after glacial recession. Black bar $=200 \mathrm{~km}$. Topography. Digital elevation model depicts a highly heterogeneous landscape; rolling grasslands in the lower elevations of the northeast and west surrounding the numerous peaks and valleys of the Rocky Mountains. Species distribution models (SDMs) predict the distribution of the species based on climate and geographic variables. The resulting model generates a map indicating areas of high and low habitat suitability based on the estimated ecological tolerance of the species. Models based on contemporary populations can be projected into past climates and species' distributions to be tracked through time. 
ent ways to represent the temporal dimensions of distributional shifts. This includes considering an approach where the temporal differences in habitat suitability from the present to historical times (e.g. 21000 вр) are translated into period-specific demographic conditions that vary over time to an approach where species distribution differences across time are represented as a single composite of past and current suitability that is translated into a spatially explicit demographic model (as modelled in Knowles \& Alvarado-Serrano 2010).

We conduct this exploration and illustrate this approach with a study of American Pikas (Ochotona princeps), a monophyletic assemblage that occurs in the northern Rocky Mountains in southwestern Canada and in northwestern United States. The American Pika, a small relative of the rabbit, is currently restricted to alpine habitats in North America's Intermountain West. The contemporary distributions of the focal populations extend from historically unglaciated regions with sky islands into post-glacially colonized areas. Fossil evidence demonstrate that this species existed at much lower elevations during the last glacial maximum (LGM) (Mead 1987; Hafner 1993; Grayson 2005), and Bayesian skyline plots of molecular data (Drummond et al. 2005) suggest that recent warming during the Holocene caused range retraction and population reduction upward into sky islands (Grayson 2005; Galbreath et al. 2009). As the continental ice sheets retreated, some of the populations migrated northwards into the previously glaciated habitats within the northern Rocky Mountains (Galbreath et al. 2009; Fig. 1). Molecular phylogenetic results corroborate these results and further reveal that gene flow during glacial periods contributed considerably to the overall pattern of diversification in pikas, under which, previously fragmented populations coalesced into regional groups (Galbreath et al. 2009). The climate changes during the LGM did not, however, facilitate extensive gene flow among populations between mountain ranges (i.e. between the adjacent Cascade ranges and the Northern Rocky Mountains; Galbreath et al. 2009, 2010).

The complex phylogeographic history, detailed knowledge of natural, fossil and glacial history and climate-mediated distribution make $O$. princeps an optimal study organism to evaluate the genetic consequences of the temporal dynamic of distributional shifts and the utility of converting SDMs into continuous carrying capacity landscapes. The life history of pikas has dramatically influenced its contemporary distribution and genetic structuring. Dispersal by pikas is limited to juveniles and is typically no $>2-3 \mathrm{~km}$; genetic results suggest that dispersal of individuals can occasionally occur over distances as great as $10 \mathrm{~km}$ (Tapper 1973; Hafner \& Sullivan 1995; Peacock 1997; Peacock \& Smith
1997). Resident adults aggressively defend territories, reducing opportunities for successful colonization by dispersers (Smith \& Ivins 1983). Owing to these factors, generally pikas exhibit low heterozygosity within populations (Smith \& Weston 1990), suggesting that inbreeding results from small effective populations. Under such scenarios, even if migration occurs at low frequencies, novel alleles are vulnerable to loss via lineage sorting owing to drift. In context of these factors, it is unrealistic to expect considerable genetic admixture between lineages even if glacial contact was extensive. We hereby test the ability of spatiotemporal demographic models to emulate these complicated histories.

Our results provide insights into key questions about integrative approaches in landscape genetics, population genetics and phylogeography. This includes (i) general issues surrounding the conversion of time-specific SDMs into simple continuous, dynamic landscapes from past to current, (ii) the utility of SDMs as proxies for habitat carrying capacities for use in demographic models as well as (iii) the contribution of the temporal dynamic on colonization history and its role in shaping genetic patterns of contemporary populations. Although SDMs are particularly useful because they can be projected forward/backward into time (Richards et al. 2007), the promise and limitations of integrative approaches that use SDMs to make species-specific predictions about patterns of genetic variation are not known. The complexity of the pika history, characterized by population distributions in the past that overlap little with contemporary populations, represents an excellent scenario for exploring the challenges in the new approaches. Moreover, as discussed, it also highlights areas in need of future development, including additional summary statistics and model validation methods that could increase the power to distinguish among the genetic signatures of different demographic models and reject (or accept) hypotheses (i.e. in an ABC framework). The exploration presented here suggests interesting implications about the history of the American Pika, although should not be confused with other estimates of biogeographic and demographic histories that are statistically more robust (e.g. in an ABC framework).

\section{Materials and methods}

\section{Species distribution models}

Species occurrence data for O. princeps (the American Pika, Ochotonidae) were collected from previous studies (Galbreath et al. 2009, 2010) and from georeferenced specimens in Cornell University Museum of Vertebrates and represented 204 unique localities across the range 
of the species. The SDM was generated in MAXENT v3.3.3e (Phillips et al. 2006) using the following parameters: random test percentage $=25$, regularization multiplier $=1$, max number of background points $=10000$, replicates $=50$, replicated run type $=$ cross-validate.

The distribution of the species was modelled for current time and projected into mid-Holocene (6000 вг) and the LGM (21 $000 \mathrm{BP})$ climate scenarios. The current SDM was initially built from the 19 standard bioclimatic variables derived through interpolation of climatic records between 1950 and 2000 from weather stations around the globe (WORLDCLIM 1.4; Hijmans et al. 2005). Environmental layers were reduced in geographic extent to an area about 30\% larger than the known distribution of the species. Bioclimatic variables were jackknifed to assess importance in the current SDM; only variables that contributed $5 \%$ or greater were included in the final model. The LGM and midHolocene climate layers were derived from the Paleoclimate Modelling Intercomparison Project Phase II (Braconnot et al. 2007). Given computational restrictions that limit the study area to 10000 unique pixels for the demographic component of the analyses, we focused on a region encompassing eastern Washington and Oregon, Idaho, western Montana and northern Wyoming in the United States and southern Saskatchewan, Alberta and British Columbia in Canada (54.9126112 ${ }^{\circ}$ $\mathrm{N},-120.883466^{\circ} \mathrm{W}$ by $\left.42.5263012^{\circ} \mathrm{N},-106.244036^{\circ} \mathrm{W}\right)$ and used a pixel resolution of 0.112611 degrees (about $12 \mathrm{~km}^{2}$ ).

As implemented here (and implicitly assumed in SDMs), we assume that the niches of the species have not changed since LGM. For the demographic modelling, we also assume that the intrinsic dispersal capabilities of the pikas have not changed over time. Given that closely related taxa utilize similar habitats (Hoffman \& Smith 2005) and all major phylogroups of $O$. princeps display little ecological divergence (Galbreath et al. 2009), there is no obvious reason that these assumptions are unreasonable for our focal taxa, which may be the case for other taxa (Wiens et al. 2009; Elith et al. 2011).

\section{Interpreting SDMs into demographic parameters}

The predicted SDMs were translated into two components for conducting the demographic simulations: (i) a friction landscape $\left(F_{l}\right)$ defining the relative ease of movement of individuals among demes and as (ii) a carrying capacity landscape $\left(K_{l}\right)$ (see also Knowles \& Alvarado-Serrano 2010). For the friction layer, SDMs models were standardized so that values ranged from zero to one and inverted (i.e. high predicted values of habitat suitability represent low friction values). In our demographic models, the number of emigrants each generation is the result of $m$ (the per-generation probability of an individual to move out of a deme) and the total number of occupants (based on local $K$ and $r$, the logistic growth parameter), whereas the spatial distribution of emigrants each generation is the result of the relative friction values of neighbouring demes. The conversion of SDMs into $K_{l}$ and $F_{l}$ was performed for each time period (i.e. for each time period a SDM was generated, and specifically, for the current, 6000 and 21000 вр).

To evaluate the utility of SDMs as proxies for developing a spatially explicit demographic model (i.e. deme-specific values specified by $F_{l}$ and $K_{l}$ ), we consider how different functions used to translate habitat suitability scores from the SDM into a demographic model affect patterns of genetic variation. In particular, we investigated three transformations: a linear, sigmoid and binary function to convert habitat suitability values from SDMs into carrying capacity values (Fig. 2). To transform that data into a sigmoid-shaped curve, we used a normal cumulative distribution function, the integral of the following equation:

$$
f(x)=\frac{1}{\sqrt{2 \pi \sigma^{2}}} \mathrm{e}^{-(x-\mu)^{2} /\left(2 \sigma^{2}\right)}
$$

where $\mu$ corresponds to the point of inflection in the curve, $\sigma$ corresponds to the slope of the curve, and $X$ is the habitat suitability score from the SDM being transformed. The lower $15 \%$ of current SDM values that correspond to observed localities was used to set $\mu$ (we also used the lower quartile), treatments called Sigmoid 1 and Sigmoid 2, respectively. This $\mu$ value was chosen so that $85 \%$ of the observed localities would lie above the inflection point and, as a result of, would possess a value of 0.5 or higher (based on a scale from 0 to 1 ) and would be classified as habitat with higher carrying capacities (Fig. 2, Supporting information). When compared to a linear transformation, using sigmoid transformation with this setting of $\mu$, a larger fraction of demes are associated with higher carrying capacities (=values $>50 \%$ of the $K_{\max }$ Figs 2 and S2, Supporting information). Both parameter values used ( $\mu$ and $\sigma$ ) were chosen to maximize the sigmoidal shape, maintaining an intermediate between binary and linear transformations. One-half of the standard deviation of the current SDM values corresponding to observed locality data was used to set $\sigma$. We explored this variable across dozens of SDMs and found this value maintained a sigmoid shaped curve and captured the ability of SDM to predict observed localities (applying a steeper slope to curves with less variation in SDM values of observed localities, JLB unpub. data). 
Species distribution models (SDMs) predict habitat suitability across the landscape

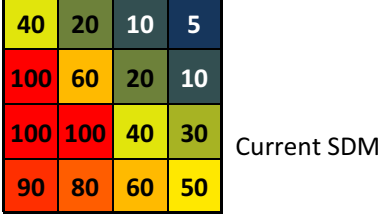

Values are transformed into different carrying capacity landscapes $\left(K_{l}\right)$

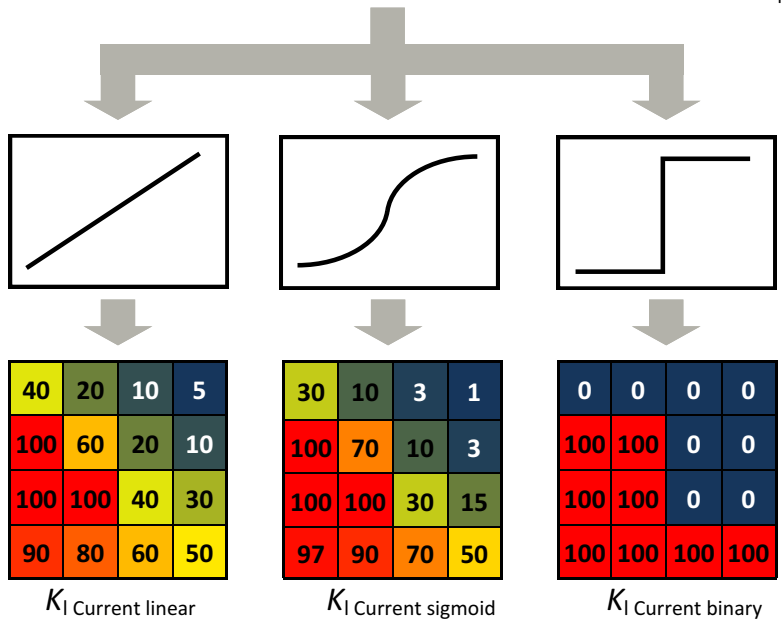

Fig. 2. Translating SDMs into carrying capacity landscapes. To investigate the impact of the defined relationship between species distribution models (SDMs) and the demographic model (specifically, $K_{l}$ ), SDMs were translated into $K_{l}$ using three different mathematic transformations: (i) a linear relationship (assuming a positive linear relationship between SDMs and carrying capacity), (ii) a sigmoid relationship (in that habitat with low suitability translates to lower carrying capacity values, however, at a threshold, carrying capacity dramatically increases and peaks), and (iii) a binary relationship (in which lower-quality habitat has a carrying capacity of zero and habitat above a threshold, has identical, high carrying capacities).

The normal cumulative distribution function was chosen over an actual sigmoid function because the parameters for $X$ directly corresponded to values obtainable from our SDM (probability values $0-1$ vs. a true sigmoidal function, values range from positive and negative values of an integer, e.g. -5 to 5). Further, the SDM suitability values that correspond to observed locality data can be used to estimate values of $\sigma$ and $\mu$ (in contrast to several transformations required by a true sigmoid function, such as the shape parameter for a sigmoid function is a positive integer and the inflection point is 0 ).

To create a binary curve, a threshold value for habitat suitability from the SDMs was considered where values greater or equal to the threshold were rescaled to the maximum carrying capacity value used in the simulations (i.e. 100, 1000 or 5000; see Table 1), whereas values less than the threshold were transformed to $k=0$. Three different thresholds were considered (Fig. 3). The first transformation, called Binary 1, excluded 15\% of observed localities with the lowest current SDM prediction values. The other two thresholds were obtained from the output of MAXENT: the minimum training presence value (MTP) and the equal training sensitivity and specificity (ETSS), called Binary 2 and Binary 3, respectively.

\section{Creating temporally continuous landscapes for demographic simulations}

The SDMs provide predictions for time-specific periods for past and current distributions that are separated by thousands of years and do not predict a continuous transition from past to current (Figs 1, 3 and S3, Supporting information). Consequently, as a basis for inferring the rate and timing of climate change history in the northern hemisphere, we used data on oxygen isotope 18/16 ratios from Greenland ice core samples, which are available at fairly fine temporal scales (Andersen et al. 2004). Specifically, to estimate demographic parameters $\left(K_{l}\right.$ and $\left.F_{l}\right)$ for time periods lacking historic climatic data for SDMs, we approximate climate change scenarios from the oxygen isotope ratio data between the three periods that bioclimatic data for SDMs is available (Fig. 3A) and this information is used to interpolate between temporally adjacent $K_{l}$ (or $F_{l}$ ) (Fig. 3B).

Several different approaches for considering how robust patterns of genetic variation were to the different ways that temporal variation might be represented in the models were considered. In addition to the spatiotemporal model described previously, we considered a model where temporal variation was summarized and represented as a static scenario for which the three SDMs (current, 6000, $21000 \mathrm{BP}$ ) were standardized from one to zero and averaged (see Knowles \& SerranoAlvarado 2010 for alternative approaches of models lacking spatiotemporal variation). This layer was then converted to a $F_{l}$ and three different $K_{l}$ using a linear transformation and a $K_{\max }$ of 100, 1000 and 5000; glacial barriers were not added to these landscapes. We also considered a model where the spatiotemporal variation between both LGM and 6000 вр SDMs and the current SDM was attenuated. This was done by averaging each model with the current SDM. We then transformed the SDMs into a $K_{l}$ using a linear transformation (hereafter called the "linear attenuated" transformation). After this, these layers were processed the same as the other SDMs.

Explicit barriers (e.g. sea level changes, rivers, ice sheets) can be integrated into each time period. In this study we incorporated glacial history (Dyke et al. 2003) into our carrying capacity landscapes $\left(K_{l}\right)$, under which, the presence of glaciers was translated into a carrying 
Table 1 Overview of how the simulated genetic data compared with the empirical data for the different scenarios

\begin{tabular}{|c|c|c|c|c|c|c|c|c|c|c|c|c|}
\hline \multicolumn{4}{|c|}{$\begin{array}{l}\text { Demographic model } \\
\text { parameters }\end{array}$} & \multicolumn{3}{|l|}{$\begin{array}{l}\text { Composite } \\
\text { statistics }\end{array}$} & \multicolumn{3}{|c|}{$\begin{array}{l}\text { Correlation } \\
\text { coefficient }\end{array}$} & \multirow[b]{2}{*}{$\begin{array}{l}\text { Total } \\
\text { rank }\end{array}$} & \multicolumn{2}{|c|}{$\begin{array}{l}\text { Transformation } \\
\text { rank score }\end{array}$} \\
\hline $\begin{array}{l}\text { Transformation } \\
\text { type }\end{array}$ & $K_{\max }$ & $m$ & $R$ & $\mathrm{x} F_{\mathrm{ST}}(\mathrm{SD})$ & x PPS (SD) & Rank & $F_{\mathrm{ST}}(r)$ & PCA $(r)$ & Rank & & Value & Rank \\
\hline Binary* & 100 & 0.01 & 0.5 & $0.638(0.099)$ & 19.533 (10.149) & 10 & 0.045 & -0.141 & 18 & 9 & 2.431 & 9 \\
\hline Binary* & 100 & 0.01 & 4 & $0.667(0.102)$ & $20.667(11.001)$ & 9 & -0.021 & -0.187 & 32 & 12 & 3.057 & 10 \\
\hline Binary* & 100 & 1 & 0.5 & $0.249(0.188)$ & $27.858(13.26)$ & 30 & 0.169 & -0.076 & 9 & 14 & 4.923 & 31 \\
\hline Binary* & 100 & 1 & 4 & $0.278(0.185)$ & 27.567 (13.204) & 27 & 0.22 & 0.001 & 4 & 11 & 4.307 & 21 \\
\hline Binary* & 1000 & 0.01 & 0.5 & $0.22(0.102)$ & 33.925 (11.904) & 42 & 0.184 & -0.17 & 15 & 27 & 4.928 & 32 \\
\hline Binary* & 1000 & 0.01 & 4 & $0.251(0.096)$ & 33.925 (12.958) & 39 & 0.199 & -0.112 & 10 & 21 & 4.587 & 26 \\
\hline Binary* & 1000 & 1 & 0.5 & $0.064(0.069)$ & $31.817(7.246)$ & 53 & -0.105 & -0.204 & 43 & 55 & 6.409 & 59 \\
\hline Binary* & 1000 & 1 & 4 & $0.056(0.051)$ & $30.992(6.594)$ & 54 & 0.026 & -0.174 & 27 & 46 & 6.087 & 55 \\
\hline Binary* & 5000 & 0.01 & 0.5 & $0.131(0.1)$ & $32.167(9.906)$ & 47 & 0.237 & -0.095 & 6 & 24 & 4.794 & 29 \\
\hline Binary* & 5000 & 0.01 & 4 & $0.131(0.102)$ & $32.592(9.982)$ & 48 & 0.223 & -0.073 & 5 & 22 & 4.795 & 30 \\
\hline Binary* & 5000 & 1 & 0.5 & $0.015(0.018)$ & $28.133(4.321)$ & 59 & -0.124 & -0.215 & 45 & 56 & 6.439 & 60 \\
\hline Binary* & 5000 & 1 & 4 & $0.012(0.014)$ & $28.65(3.953)$ & 60 & -0.053 & -0.105 & 26 & 48 & 5.944 & 52 \\
\hline Linear* & 50 & 1 & 4 & $0.717(0.15)$ & $11.85(10.426)$ & 2 & 0.355 & -0.2 & 7 & 3 & 1.792 & 2 \\
\hline Linear* & 100 & 0.01 & 0.5 & $0.884(0.054)$ & $33.642(23.662)$ & 25 & -0.017 & -0.397 & 54 & 39 & 5.586 & 46 \\
\hline Linear* & 100 & 0.01 & 4 & $0.882(0.057)$ & $31.4(25.514)$ & 23 & 0.114 & -0.524 & 57 & 38 & 5.22 & 37 \\
\hline Linear* & 100 & 1 & 0.5 & $0.573(0.19)$ & $12.617(8.082)$ & 6 & 0.402 & -0.039 & 1 & 1 & 1.952 & 5 \\
\hline Linear* & 100 & 1 & 4 & $0.68(0.177)$ & 13.9 (10.984) & 1 & 0.321 & -0.27 & 14 & 5 & 1.92 & 3 \\
\hline Linear* & 1000 & 0.01 & 0.5 & $0.513(0.16)$ & 26.685 (16.105) & 13 & -0.047 & -0.27 & 44 & 25 & 3.784 & 17 \\
\hline Linear* & 1000 & 0.01 & 4 & $0.531(0.158)$ & 28.944 (17.929) & 15 & 0.093 & -0.226 & 25 & 16 & 3.536 & 14 \\
\hline Linear* & 1000 & 1 & 0.5 & $0.148(0.091)$ & $28.75(8.295)$ & 41 & 0.129 & -0.218 & 19 & 33 & 5.052 & 35 \\
\hline Linear* & 1000 & 1 & 4 & $0.179(0.122)$ & $29.343(10.075)$ & 37 & 0.052 & -0.317 & 39 & 42 & 5.317 & 41 \\
\hline Linear* & 5000 & 0.01 & 0.5 & $0.261(0.13)$ & $33.208(15.03)$ & 34 & 0.015 & -0.158 & 24 & 31 & 5.205 & 36 \\
\hline Linear* & 5000 & 0.01 & 4 & $0.256(0.127)$ & 32.975 (14.007) & 35 & -0.127 & -0.31 & 55 & 53 & 5.73 & 48 \\
\hline Linear* & 5000 & 1 & 0.5 & $0.045(0.034)$ & $29.642(4.577)$ & 56 & 0.005 & -0.287 & 40 & 54 & 5.796 & 49 \\
\hline Linear* & 5000 & 1 & 4 & $0.052(0.04)$ & $29.575(6.122)$ & 50 & -0.088 & -0.348 & 56 & 58 & 6.082 & 54 \\
\hline Linear Attn.* & 100 & 0.01 & 4 & $0.861(0.075)$ & $22.425(20.103)$ & 12 & 0.15 & -0.409 & 41 & 23 & 3.772 & 16 \\
\hline Linear Attn.* & 100 & 0.1 & 0.5 & $0.729(0.137)$ & 14.55 (11.754) & 5 & 0.325 & -0.26 & 13 & 7 & 2.014 & 7 \\
\hline Linear Attn.* & 100 & 1 & 0.5 & $0.565(0.199)$ & $12.792(9.346)$ & 8 & 0.43 & -0.117 & 2 & 2 & 1.95 & 4 \\
\hline Linear Attn.* & 100 & 1 & 4 & $0.581(0.192)$ & $13.483(8.553)$ & 7 & 0.344 & -0.217 & 8 & 6 & 2.114 & 8 \\
\hline Linear Attn.* & 1000 & 0.01 & 0.5 & $0.487(0.129)$ & $26.742(14.203)$ & 17 & 0.048 & -0.166 & 21 & 15 & 3.697 & 15 \\
\hline Linear Attn.* & 1000 & 0.01 & 4 & $0.502(0.148)$ & $26.583(16.389)$ & 14 & 0.049 & -0.197 & 28 & 17 & 3.535 & 13 \\
\hline Linear Attn.* & 1000 & 1 & 0.5 & $0.159(0.116)$ & $28.192(8.777)$ & 38 & 0.068 & -0.267 & 33 & 37 & 5.255 & 38 \\
\hline Linear Attn.* & 1000 & 1 & 4 & $0.159(0.108)$ & $29.55(8.951)$ & 40 & 0.022 & -0.386 & 51 & 51 & 5.583 & 44 \\
\hline Linear Attn.* & 5000 & 0.01 & 0.5 & $0.26(0.131)$ & $32.783(14.086)$ & 33 & 0.048 & -0.168 & 22 & 28 & 4.977 & 33 \\
\hline Linear Attn. ${ }^{*}$ & 5000 & 0.01 & 4 & $0.263(0.132)$ & 33.533 (15.095) & 36 & -0.061 & -0.239 & 42 & 41 & 5.435 & 42 \\
\hline Linear Attn.* & 5000 & 1 & 0.5 & $0.04(0.032)$ & $29.075(5.252)$ & 55 & 0.005 & -0.208 & 30 & 49 & 5.835 & 50 \\
\hline Linear Attn.* & 5000 & 1 & 4 & $0.047(0.043)$ & $29.725(6.036)$ & 52 & -0.103 & -0.374 & 59 & 59 & 6.186 & 56 \\
\hline Sigmoid v2 * & 5000 & 1 & 4 & $0.428(0.244)$ & $31.5(23.771)$ & 24 & -0.074 & -0.389 & 58 & 45 & 4.771 & 28 \\
\hline Sigmoid v1* & 100 & 0.1 & 4 & $0.829(0.122)$ & $18.133(23.07)$ & 11 & 0.237 & -0.55 & 48 & 20 & 3.452 & 11 \\
\hline Sigmoid v1* & 100 & 1 & 0.5 & $0.766(0.146)$ & $11.3(8.658)$ & 4 & 0.457 & -0.415 & 17 & 8 & 1.958 & 6 \\
\hline Sigmoid v1* & 100 & 1 & 4 & $0.755(0.152)$ & $11.433(8)$ & 3 & 0.43 & -0.313 & 11 & 4 & 1.716 & 1 \\
\hline Sigmoid v1* & 1000 & 0.1 & 4 & $0.458(0.21)$ & $29.783(20.664)$ & 21 & -0.059 & -0.347 & 53 & 35 & 4.468 & 24 \\
\hline Sigmoid v1* & 1000 & 1 & 0.5 & $0.495(0.237)$ & 25.883 (19.184) & 16 & 0.122 & -0.317 & 34 & 19 & 3.484 & 12 \\
\hline Sigmoid v1* & 1000 & 1 & 4 & $0.478(0.232)$ & $28.808(20.874)$ & 19 & 0.026 & -0.395 & 52 & 32 & 4.337 & 23 \\
\hline Sigmoid v1* & 5000 & 0.01 & 0.5 & $0.324(0.188)$ & 32.375 (18.136) & 28 & -0.018 & -0.184 & 29 & 29 & 4.646 & 27 \\
\hline Sigmoid v1* & 5000 & 0.01 & 4 & $0.332(0.203)$ & $32.383(19.594)$ & 29 & -0.018 & -0.189 & 31 & 30 & 4.581 & 25 \\
\hline Sigmoid v1* & 5000 & 1 & 0.5 & $0.265(0.183)$ & 32.275 (17.905) & 32 & 0.037 & -0.268 & 36 & 36 & 5.018 & 34 \\
\hline Sigmoid v1* & 5000 & 1 & 4 & $0.282(0.198)$ & 32.175 (18.592) & 31 & -0.048 & -0.303 & 47 & 44 & 5.288 & 39 \\
\hline Static ${ }^{*}$ & 100 & 0.01 & 0.5 & $0.887(0.059)$ & $54.108(46.048)$ & 49 & 0.283 & -0.268 & 16 & 34 & 5.571 & 43 \\
\hline Static ${ }^{*}$ & 100 & 0.01 & 4 & $0.852(0.07)$ & $59.85(46.398)$ & 57 & 0.154 & -0.257 & 23 & 43 & 5.91 & 51 \\
\hline Static* & 100 & 1 & 0.5 & $0.512(0.189)$ & $42.15(23.222)$ & 26 & 0.358 & -0.089 & 3 & 10 & 4.202 & 20 \\
\hline Static ${ }^{*}$ & 100 & 1 & 4 & $0.531(0.206)$ & 35.9 (20.89) & 22 & 0.273 & -0.19 & 12 & 13 & 3.824 & 18 \\
\hline
\end{tabular}


Table 1 Continued

\begin{tabular}{|c|c|c|c|c|c|c|c|c|c|c|c|c|}
\hline \multicolumn{4}{|c|}{$\begin{array}{l}\text { Demographic model } \\
\text { parameters }\end{array}$} & \multicolumn{3}{|l|}{$\begin{array}{l}\text { Composite } \\
\text { statistics }\end{array}$} & \multicolumn{3}{|c|}{$\begin{array}{l}\text { Correlation } \\
\text { coefficient }\end{array}$} & \multirow[b]{2}{*}{$\begin{array}{l}\text { Total } \\
\text { rank }\end{array}$} & \multicolumn{2}{|c|}{$\begin{array}{l}\text { Transformation } \\
\text { rank score }\end{array}$} \\
\hline $\begin{array}{l}\text { Transformation } \\
\text { type }\end{array}$ & $K_{\max }$ & $m$ & $R$ & $\mathrm{x} F_{\mathrm{ST}}(\mathrm{SD})$ & x PPS (SD) & Rank & $F_{\mathrm{ST}}(r)$ & PCA $(r)$ & Rank & & Value & Rank \\
\hline$\overline{\text { Static }}{ }^{*}$ & 1000 & 0.01 & 0.5 & $0.457(0.147)$ & 29.133 (15.701) & 20 & 0.009 & -0.265 & 38 & 26 & 4.333 & 22 \\
\hline Static ${ }^{*}$ & 1000 & 0.01 & 4 & $0.474(0.153)$ & 29.075 (14.406) & 18 & 0.001 & -0.114 & 20 & 18 & 3.978 & 19 \\
\hline Static ${ }^{*}$ & 1000 & 1 & 0.5 & $0.114(0.096)$ & $27.367(6.2)$ & 44 & 0.042 & -0.272 & 35 & 40 & 5.317 & 40 \\
\hline Static* & 1000 & 1 & 4 & $0.127(0.098)$ & $28.033(6.995)$ & 43 & 0.011 & -0.373 & 49 & 52 & 5.585 & 45 \\
\hline Static ${ }^{*}$ & 5000 & 0.01 & 0.5 & $0.172(0.088)$ & $32.6(11.37)$ & 46 & -0.015 & -0.232 & 37 & 47 & 5.702 & 47 \\
\hline Static* & 5000 & 0.01 & 4 & $0.191(0.102)$ & $33.367(12.01)$ & 45 & -0.1 & -0.242 & 46 & 50 & 5.953 & 53 \\
\hline Static* & 5000 & 1 & 0.5 & $0.024(0.028)$ & $28.667(4.736)$ & 58 & -0.201 & -0.295 & 60 & 60 & 6.213 & 57 \\
\hline Static* & 5000 & 1 & 4 & $0.038(0.049)$ & $28.908(5.125)$ & 51 & -0.162 & -0.229 & 50 & 57 & 6.262 & 58 \\
\hline
\end{tabular}

Average summary statistics across all populations. To evaluate how similar the simulated values were to those observed in the genetic data, we considered a number of summaries of genetic variation calculated as averages across all populations: average $F_{\mathrm{ST}}$ and the average number of private polymorphic sites (PPS) calculated across the different populations are presented (see Fig. 6).

The observed mean and standard deviation for $F_{\mathrm{ST}}$ and PPS was $0.682 \pm 0.183$ and $7.25 \pm 6.69$, respectively. Correlation coefficient between observed and simulated data sets. To summarize the patterns of genetic divergence across populations (as opposed to an average $F_{\mathrm{ST}}$ ) two correlation coefficients were considered between the observed and simulated data: (i) a pairwise $F_{\mathrm{ST}}$ distance matrix among populations and (ii) a standardized principal component analysis (SPCA) of the mean number of PPS, mean number of alleles over loci, mean $F_{\mathrm{ST}}$, mean heterozygosity and mean pairwise differences (see Figs S4 and S5, Supporting information). Ranks: the different demographic models were then ranked to assess the total ability of the model to emulate the observed genetic data. Total Rank: Lastly, we summed composite scores (values ranked for both types of summary statistics) and ranked them. This rank represented the total model fit to the empirical data. Transformation Rank Score: The pika populations exhibit a complicated biogeographic history, to evaluate the general correspondence between the simulated data under different demographic models and more simple biogeographic histories; we selected two subsets of the pika data set displaying different levels of isolation-by-distance (Figs S4 and S5, Supporting information). We then calculated the same summary statistics and ranks measured for the entire data set for each subset. To evaluate how different transformations used to convert deme-specific values from the species distribution models (SDMs) into carrying capacities, $K_{l}$, impacted patterns of genetic variation, and consequently, the match between simulated and empirical data sets, a "Transformation Rank Score" was created. This consisted of ranking the sum of the composite scores from the complete data set and the two subsets (weighing the subsets by $50 \%$ and the complete data set by $100 \%$, see Fig. 7) Linear* refers to transformations in which the SDM was multiplied by $K_{\max }$. Linear Attn* refers to a transformation where the spatiotemporal variation between both LGM and 6000 вр SDMs and the current SDM was attenuated. In all other capacities Linear Attn* it is identical to Linear*. Sigmoid v1* and Sigmoid v2* refers to transformations based on a normal distribution function. The inflection point is based on the lower $15 \%$ and lower quartile current SDM values for observed localities, respectively. Binary* refers to transformations that converted the lower 15\% current SDM values to zero and everything above to $K_{\max }$. Static* refers to transformations where species distribution differences across time are represented as a single composite of past and current suitability. If data were not presented for a transformation, the simulations were performed, however, did not result in colonization of all populations.

capacity value of one, regardless of corresponding value from SDMs.

\section{Demographic and genetic simulations}

A demographic colonization process was simulated based on migration, growth and density limitations imposed by the environment (the $F_{l}$ and $K_{l}$ landscapes) using a modified version of SPLATCHE 2 (Ray et al. 2010). These time forward simulations were performed from past to current conditions, with the colonization of contemporary populations dependent upon the demographic and environmental parameters (Figs 4 and 5). To examine the genetic consequences of the distributional shifts, coalescent genealogies parameterized by the spatially explicit demographic conditions were simulated and genes were sampled for focal populations (Fig. 4; for an overview of data flow and input parameters, Excoffier et al. 2000; Currat et al. 2004; Knowles \& Serrano-Alvarado 2010; Ray et al. 2010).

For the genetic simulations we focused on conditions that correspond to empirical data sets with both nuclear and mitochondrial genetic data available (Galbreath et al. 2009, 2010). Specifically, all the simulated genetic data matched the empirical data with respect to the number of individuals and spatial distribution, the number of loci sequenced, number of base pairs per locus and corresponding model of nucleotide evolution. 


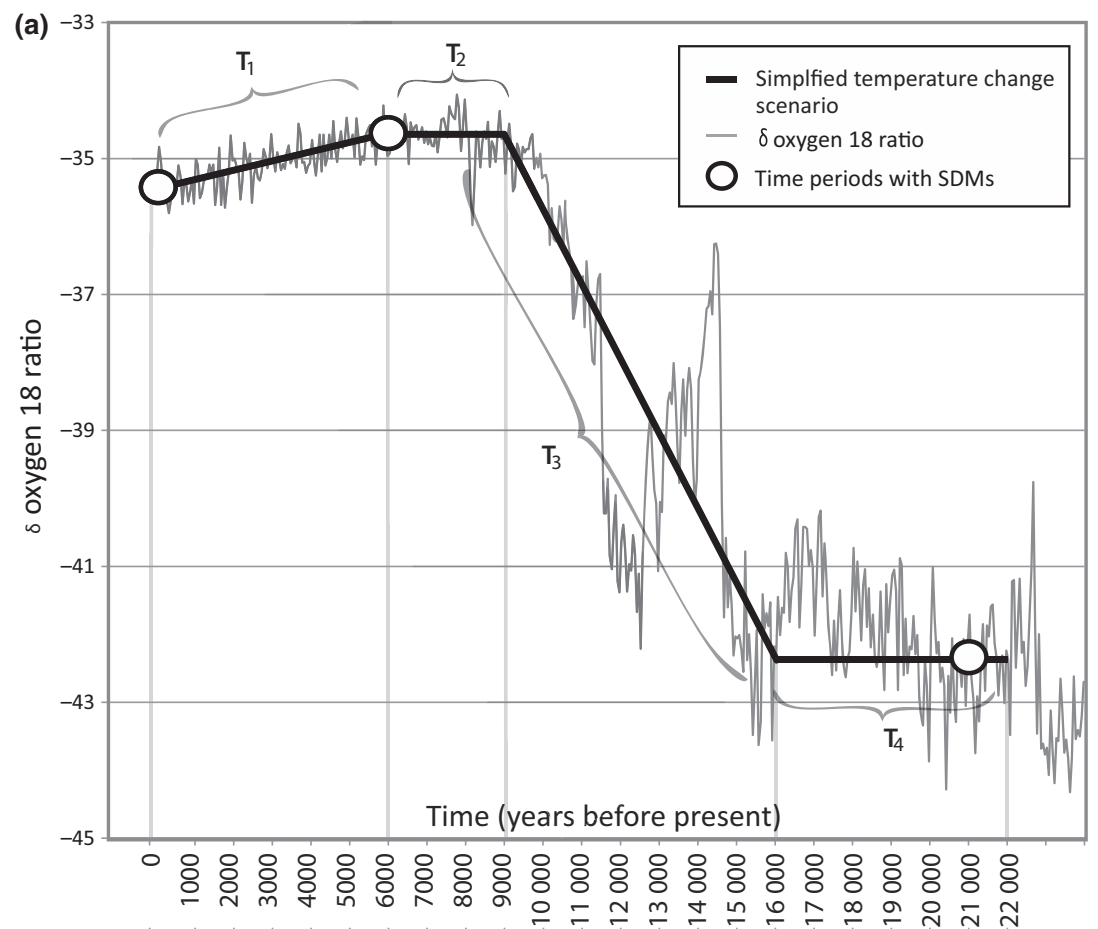

(b)

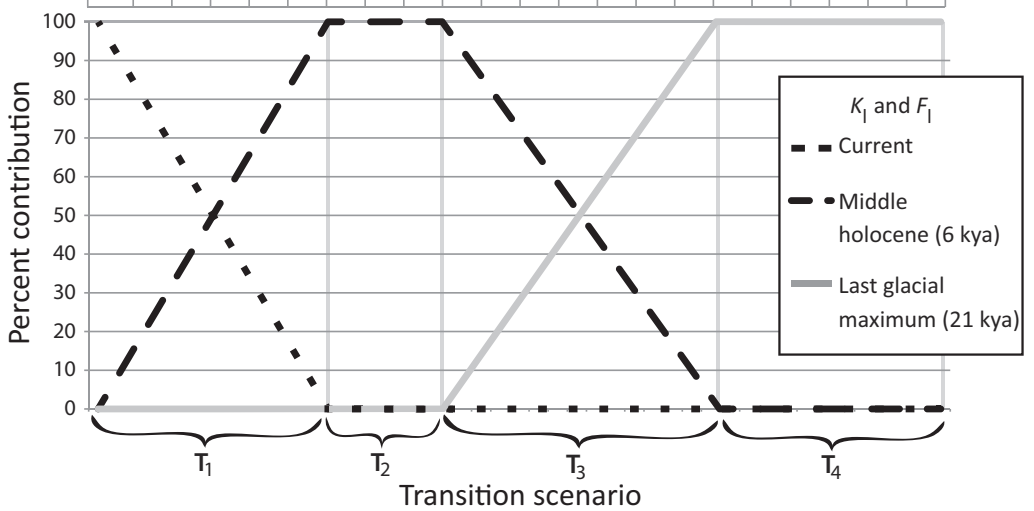

Fig. 3. (A) Post-LGM temperature change scenario for the northern hemisphere based on oxygen isotope 18/16 ratios from Greenland ice core samples (Andersen et al. 2004). It is estimated that each one part per thousand change in $\delta^{18} \mathrm{O}$ represents roughly a $1.5-2{ }^{\circ} \mathrm{C}$ change in sea surface temperatures (Veizer et al. 2000). These data were not used to determine the exact temperature transition scenarios, but rather, the rate of climate change during the intervening periods for which SDMs can be created (marked with the open circles). The rate of climate change inferred from the oxygen-isotope data was used to specify SDMs, which was then transformed into corresponding $F_{l}$ and $K_{l}$. To simplify extrapolation, the rate of climate change was divided into four periods (or transitions between time periods with SDMs) as defined by : (1) if the rate of climate change (i.e. slope) equalled zero adjacent to a SDM, then that SDM contributed $100 \%$ to the time slice $\left(T_{2}\right.$ and $\left.T_{4}\right)$ or (2) if the simplified temperature change slope was nonzero, a linear transition between the two SDMs occurred $\left(T_{1}\right.$ and $\left.T_{3}\right)$ and the rate at which the two models were mixed was determined by the time between SDMs. (B) Contribution of $K_{l}$ and $F_{l}$ to environmental time-slices. The four transition scenarios were created to reflect the rate of climate change relative to periods for which $F_{l}$ and $K_{l}$ can be estimated directly from periods with SDMs (marked with the open circles in Fig. 3A). In this study, we generated time-slices at 500 year intervals. In addition to SDM information, we incorporated glacial history into our carrying capacity landscapes $\left(K_{l}\right)$, under which, the presence of glaciers was translated into a carrying capacity value of one, regardless of corresponding value from SDMs.

Consequently, the genetic data set simulated for this study corresponded to the empirical data that consisted of $1632 \mathrm{bp}$ mtDNA (cytochrome $b$ gene and D-loop) and $1087 \mathrm{bp}$ of nDNA (561 bp of protein kinase C iota and $526 \mathrm{bp}$ mast cell growth factor intron) for 60 individuals from 12 populations (Galbreath et al. 2009, 2010; see Appendix S1 and Fig. S1, Supporting information for localities and number of individuals).

Genetic data were simulated using a sequence transition-transversion rate estimated in MACCLADE 4 


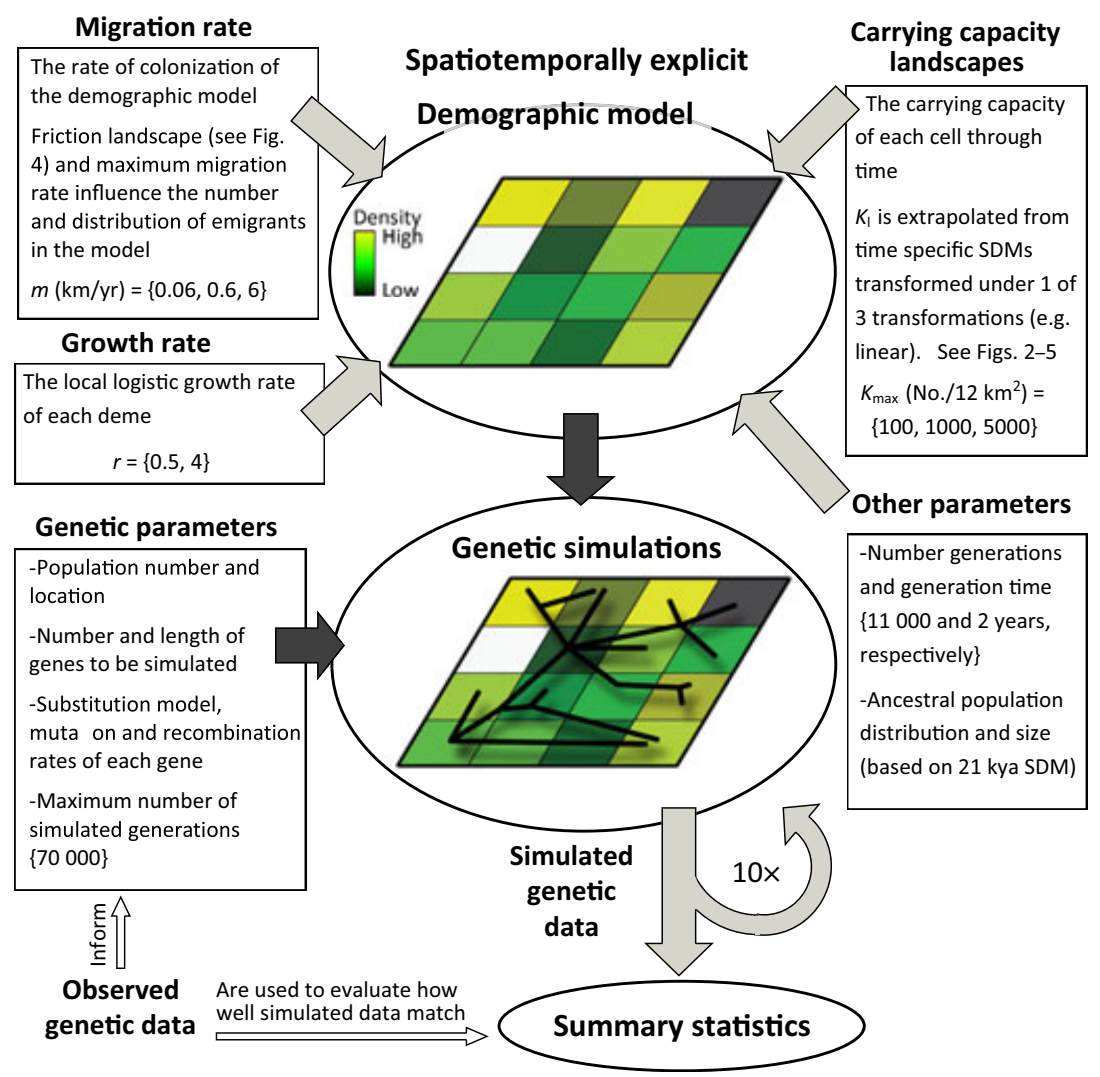

Fig. 4. Overview of parameters input into demographic and genetic simulations and summarization of genetic data from simulations that is compared with empirical genetic data. A demographic colonization process was simulated based on migration rate, growth rate and density limitations imposed by the environment $\left(F_{l}\right.$ and $\left.K_{l}\right)$. The patterns of genetic diversity were then estimated from nucleotide data sets simulated from the coalescent simulations that were informed from the demographic colonization history. Replicate genetic data sets were generated for each scenario (i.e. each set of demographic parameters and transformation types used to convert information from the SDM into the demographic model; for a summary of scenarios see Table 1).

(Maddison \& Maddison 2005) using the entire data set from Galbreath et al. 2009; rates of recombination were set to $0.537 \mathrm{cM} / \mathrm{Mb}$ for $\mathrm{nDNA}$ and zero for mtDNA based on recombination rates of other Anagaloids (Jensen-Seaman et al. 2004; Dumont \& Payseur 2008). For the genetic simulations, we used a mtDNA mutation rate of $4.8 \%$ per Myr estimated specifically for the pika data set (Galbreath et al. 2009) and nDNA mutation rate estimated for mammals of $0.22 \%$ per Myr (Kumar \& Subramanian 2002). Although the latter has been criticized owing to dramatic differences in generation times observed in mammals (Sims et al. 2009), the generation times of pika are intermediate among mammals, making it less likely that spurious estimates are associated with extreme generation times.

A range of demographic parameter values and environmental characteristics were simulated so that their effects on the patterns of genetic variation, and specifically, on the levels of genetic differentiation among populations (where the populations of interest across the landscape correspond to those in the empirical data) could be compared. Specifically, the following parameters were considered in the demographic simulations: 11000 generations ( 1 generation $=2$ years) (Peacock 1997), growth rates between 0.5 and 4 proportional increase/gen. (see Table 1, values from Brant 1989) and migration rates between 0.06 and $6 \mathrm{~km} /$ year (Tapper 1973; Hafner \& Sullivan 1995; Peacock 1997; Peacock \& Smith 1997). The narrow habitat preferences of pikas, small spatial scale of population surveys and the extreme habitat heterogeneity of the study area made it difficult estimate a precise maximum carrying capacity of the landscape and because of, we evaluated a broad range of biologically relevant carrying capacity parameters (Millar 1974; Southwick et al. 1986; Smith \& Hanski 1998). Initial population densities in the demographic model were input from a modified LGM $K_{l}$ (with a linear transformed with $K_{\max }$ equal to that of the $K_{l}$ in the simulation) where the presence of glaciers and SDM values below 0.600 were converted to zero. This initial distribution was corroborated by LGM fossil data with populations overlapping with fossil distributions (Mead 


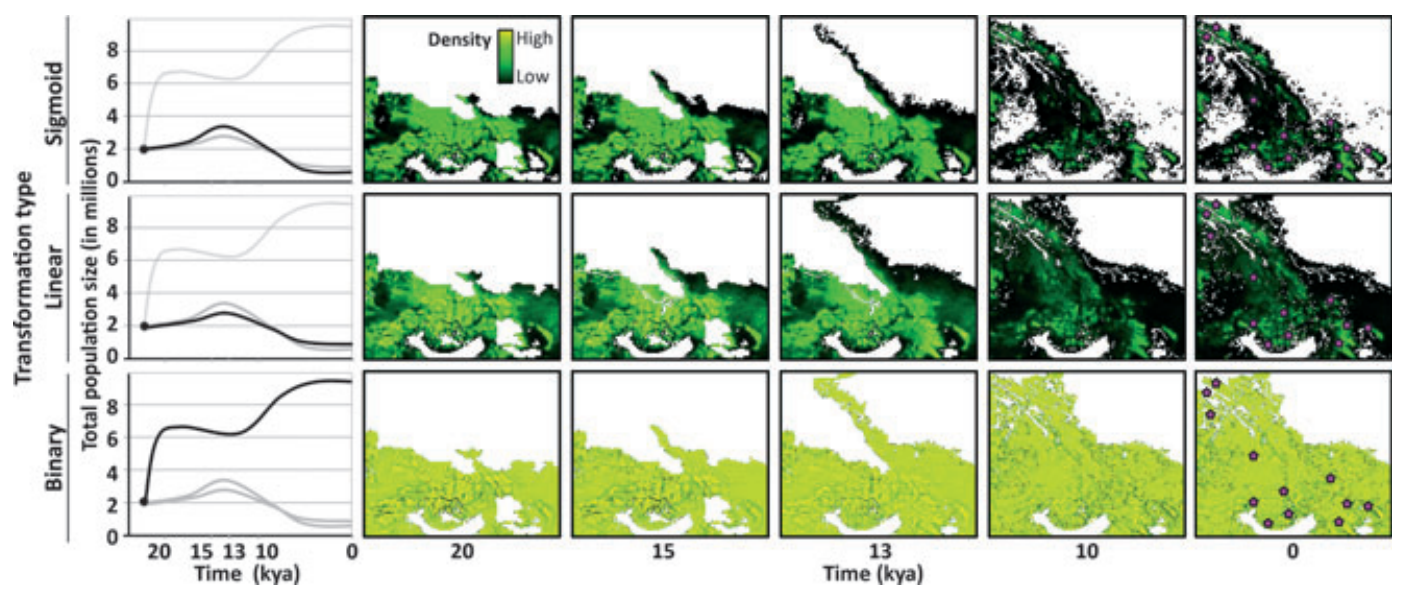

Fig. 5. The impact of different transformation types (left) on the distribution of population sizes across time (on the right). Transformation type (left). Population density at the start of the simulations at 22000 вр (depicted by a black dot) was c. 2 million and light grey lines compare the population sizes over time for a given transformation (shown in black) with the other two transformations. Distributions of populations through time (right). Bright yellow colours depict higher population densities and darker colours represent lower densities. Areas unoccupied are white. Pink stars at Time $=0$ represent the contemporary locations of the 12 focal populations. The data shown were simulated with: $K_{\max }=1000, m=1, r=4$ and an initial population corresponding to $K_{\max }$.

1987; Hafner 1993; Grayson 2005). The value of 0.600 was chosen because it is the median of current SDM values of the 204 observation localities. Although this value is somewhat arbitrary, we felt this was a conservative interpretation of the LGM SDMs, using information relevant to contemporary and LGM fossil populations to focus the initial colonization on historic populations with the highest prediction values.

For each set of demographic conditions (summarized in Table 1), ten replicate genetic simulations were performed with a maximum of 70000 generations per genetic simulation (not to be confused with the 11000 used in the demographic models, which spans the period from the present to the LGM, $22000 \mathrm{BP})$. The maximum of 70000 generations per genetic simulation ensured coalescence of all genes in the study. Only the results from demographic simulations in which all 12 focal populations are colonized are reported (i.e. under some scenarios the expansion process did not result in migrants colonizing all the focal populations).

\section{Evaluating the impact of demographic model parameters}

A MANOVA, with all summary statistics used as dependent variables, was used to evaluate how patterns of genetic variation were influenced by the individual parameters (e.g. transformation type, $K_{\max }, r, m$ ). Summary statistics that did not display homogeneity of variance were arcsine transformed before analysis. If this failed to achieve homogeneity of variance, the impact of the parameter on patterns of genetic variation was evaluated using a nonparametric Kruskal-Wallis $H$ test and Games-Howell post-hoc test, analysing each untransformed summary statistic independently. Significance in the MANOVA was determined using Wilks' Lambda and Games-Howell post hoc tests. Because of a limited number of replicates that resulted in colonization of the targeted populations for some parameter values (specifically, $K_{\max }=50, m=0.1$ or transformation type $=$ Sigmoid v2), these were not included in the MANOVA.

To assess pairwise differences among the types of transformations used to convert information from the SDMs into a demographic model and to control for variation added by $m$ and $K_{\max }$ (see Results for justification), two additional parameter values for $r$ were used in the simulations $(r=1$ and $r=2)$ for the transformations at $K_{\max }=100$ and $m=1$. This increased sample sizes within simulations, thereby increasing the power for statistical analysis for these demographic settings (see Table S1, Supporting information for summary statistics of this data). Comparisons of group ranks scores were performed in Mann-Whitney $U$ test. Analyses were performed in SPSS $\mathrm{v}$ 17.0.

\section{Measuring spatiotemporal variation and habitat heterogeneity}

Different transformation types have the effect of varying the extent of spatiotemporal variation and habitat heterogeneity. Consequently, we quantified the spatiotemporal variation and spatial heterogeneity of carrying capacities for each of the transformation types. To 
characterize spatiotemporal variation, standard deviation of each cell was calculated among the three time periods: current (0 BP), 6000 and $21000 \mathrm{BP}$ (periods which SDMs were created) and each value was divided by the maximum standard deviation possible. This resulted in values between zero and one, with one equalling the maximum temporal change per cell (equating to the transition directly from the minimum to maximum $K_{\max }$ values or vice versa). A global value was measured by calculating the mean of the standardized SD values for all the cells in the study area.

To quantify spatial heterogeneity we evaluated two different aspects of heterogeneity: overall variation and the level of spatial autocorrelation. Overall variation was measured by calculating the coefficient of variation (CV) for the study area for the current (0 Bг), 6000 вр and LGM (21 000 вг) time periods. A global CV was calculated by weighting each $\mathrm{CV}$ by the period of time that it contributed $>50 \%$ to the carrying capacity layers (Fig. 3B) and all were summed. Higher CV values correspond to higher levels habitat variation. To measure the magnitude of global spatial autocorrelation, we calculated Morin's I of the same three time periods (current, 6000 and 21000 вр). The global Morin's I was calculated by weighting each time period the same as done in the CV. In general, a Moran's Index value near +1.0 indicate strong clustering of habitat values while an index value near -1.0 indicate highly dispersed habitat values and values near zero indicate a random spatial distribution of habitat values.

\section{Evaluating whether scenarios generate patterns of genetic variation observed in the empirical data}

Summary of average population genetic patterns. To evaluate how similar the simulated values were to those observed in the genetic data, we considered a number of summaries of genetic variation calculated as averages across all populations: the number of pairwise nucleotide differences $(\pi)$, heterozygosity, number of segregating sites, number of private polymorphic sites (PPS, the number of polymorphic sites unique to each population) and $F_{\mathrm{ST}}$ (based on genetic distances from a Tamura \& Nei substitution model). All genetic calculations were performed in ARLSUMSTAT and ARLEQUIN 3.5.1.2 (Excoffier \& Lischer 2010). Because of significant correlations among some summary statistics (based on Pearson correlation coefficients that were $>0.90$ ), only those statistics that extract independent information were used to summarize how similar simulated data sets were to the observed genetic data. Specifically, only results from the average $F_{\mathrm{ST}}$ and the average PPS calculated across the different populations are presented (Table 1).
Distribution of genetic variation across populations. To summarize the patterns of genetic divergence across populations of each simulation, the match between the simulated and observed genetic data for each population (as measured by different summary statistics) was summarized as a correlation. We calculated two correlation coefficients between observed and simulated data: (i) a pairwise $F_{\mathrm{ST}}$ distance matrix among populations and (ii) a standardized principal component analysis (SPCA) of the following summary statistics measured within each population: mean number of PPS, mean number of alleles over loci, mean $F_{\mathrm{ST}}$, mean heterozygosity and mean pairwise differences (Table 1). The SPCA consisted of correlations to several eigenvectors; each vector was weighted by their loading and summed to create a composite correlation. Both correlations (SPCA and pairwise $F_{\mathrm{ST}}$ ) focus on the distribution of genetic variation across populations (i.e. the geographic distribution of genetic variation) rather than the actual values at each population. The higher the correlation coefficient, the more similar the distribution of genetic variation across populations between the simulated and observed data sets.

A common method to calculate summary statistics for Approximate Bayesian computations (ABC) uses a linear regression (Beaumont et al. 2002) or a general linear model (GLM, Leuenberger \& Wegmann 2010) to guide retention parameters that produce summary statistics equal to the observed values. In this study, each simulation required extensive data preparation and our ability to perform thousands of simulations is limited. Because of this, we were less interested in the form (or shape) of the relationship of parameters to summary statistics, a focus of linear regressions and GLMs, and were more focused on the strength of a relationship between two data sets, which is better illustrated by a correlation coefficient and used in this study (see also Ray et al. 2005).

Relative differences in the match between simulated and observed genetic data across models. The different scenarios (i.e. different models with different transformations and demographic parameters) were ranked according to the similarity between the simulated and observed genetic data. We calculated the average difference between the observed and simulated population summary statistics (both means and standard deviations of values) and these values were standardized from 0 to 1 (where values of zero represent the value closest the observed value). All the standardized values from the different tests of fit were weighted and summed; mean values contributed to $90 \%$ and standard deviations contributed $10 \%$ to a composite score (value to be ranked). Under this scenario this rank represented how well the simulations matched genetic values of the observed data set. 
The correlation coefficients were standardized from 0 to 1 (inverting the coefficients, so that values of zero corresponded to the highest coefficients) and then summed and ranked. This rank represented how well the simulations matched the observed pattern of divergences among populations. Lastly, we summed composite scores (values ranked for both types of summary statistics) and ranked them. This rank represented the total correspondence between the data simulated under a particular model and the empirical data (in Table 1 as "Total Rank").

\section{Considering more simplified biogeographic scenarios}

The pika populations exhibit a complicated biogeographic history with many geographically proximal populations displaying high $F_{\mathrm{ST}}$ whereas geographically distant populations display low $F_{\mathrm{ST}}$ values. Because of the complicated biogeographic history of this species, we considered two subsets of the pika data set to evaluate how well each demographic model captured different processes. The first subset (herein referred to as Subset 1; consisting of populations Grande Cache, McBride, Roman Nose and Neihart, see Supporting information and Fig. S1, Supporting information) exhibited strong evidence of isolation-by-distance (IBD) (Mantel test: $r=0.903, P<0.001$ ). The other subset (Subset 2; consisting of populations Raft Mountain, McBride, Roman Nose and Darby) displayed no evidence of IBD (Mantel test: $r=-0.173, P \approx 0.60$ ). We calculated the same summary statistics and ranks measured as for the entire data set described previously. In addition, we created a "Transformation Rank Score" that consisted of the sum of the composite scores from the complete data set and the two subsets (weighted the subsets by $50 \%$ and the complete data set by 100\%) and then ranked this score. The "Transformation Rank Score" does not necessarily indicate the best overall model of pika phylogeography and the subset populations are weighted more in the final score than others (the best overall models for pikas is indicated by the "Total Rank" of the complete data set). This rank does, however, better classify the ability of each model to emulate both complicated and simple biogeographic histories and the potential utility of the performed transformations in a wide array of future studies.

\section{Results and discussion}

\section{Impact of transformation type and demographic model parameters on genetic variation}

Transformation type (MAnOva: $F=2.850, \mathrm{df}=16, P<$ 0.001 ), maximum carrying capacity (Kruskal-Wallis. x $F_{\mathrm{ST}}: \chi=30.824, \mathrm{df}=2, P<0.001 ; \mathrm{x}$ PPS: $\chi=8.075$, $\mathrm{df}=2, P=0.018 ; \rho F_{\mathrm{ST}}: \chi=25.842, \mathrm{df}=2, P<0.001 ; \rho$ PCA: $\chi=0.295, \mathrm{df}=2, P=0.863$ ) and migration rate (MANOva: $F=14.388, \mathrm{df}=4, P<0.001$ ) (Figs 6,7 , S4 and S5, Supporting information) all had a significant impact on the patterns of genetic variation, as captured from the different summary statistics used to characterize the genetic data (see Appendices S2 and S3, Supporting information for videos of different parameters values and their effect on the demographic models). Growth rates produced more attenuated effects on summary statistics and were probably overshadowed by the other parameters (Figs 6, S4 and S5, Supporting information; MANOVA: $F=0.903, \mathrm{df}=4, P=0.619)$. However, growth rates were particularly important in models with low $K_{\max }$ and $m$ values. Under such scenarios, models with higher growth rates were key to the persistence of local demes in the demographic simulations and resulted in successful colonization of all focal populations. In general, lower values of $K_{\max }$, and $m$ produced higher $F_{\mathrm{ST}}$ values, lower PPS and higher variance of composite statistics among populations. The significant effect of specific demographic parameters (e.g. $k$ and $m$ ) on patterns of genetic variation highlights that species-specific life history traits may have an important influence on phylogeographic patterns (see also Knowles and SerranoAlvarado 2010). Our findings here also demonstrate that the transformation type (i.e. how habitat suitability is converted into demographic parameters) has a significant effect on patterns of genetic variation and suggests such integrative analyses will need to include the transformation itself as an addition parameter to be estimated. If such parameter rich models exceed the information content of the data (e.g. Gelman \& Meng 1996; Neuenschwander et al. 2008; Verdu et al. 2009), at minimum, different transformations should be considered and the sensitivity of the results explored. Another consequence of applying transformations to $K_{l}$ is a general reduction in the collinearity between friction and carrying capacity landscapes (parameters based on the same SDM, Table S2, Supporting information).

Several scenarios did not result in the colonization of all populations, especially for certain transformation types, such as Binary v2, Binary v3 and Sigmoid v2. For such transformations, a large proportion of the habitat was converted into low carrying capacity values. As a result, the demographic model shifted from a regional colonization model mediated by $K_{l}$ and $F_{l}$ to a local extinction model with small regional demes with no path of colonization to suitable adjacent habitats. This phenomenon was also common in situations where SDM values were transformed into too low carrying capacity values for low values of $K_{\max }$. All discussions 

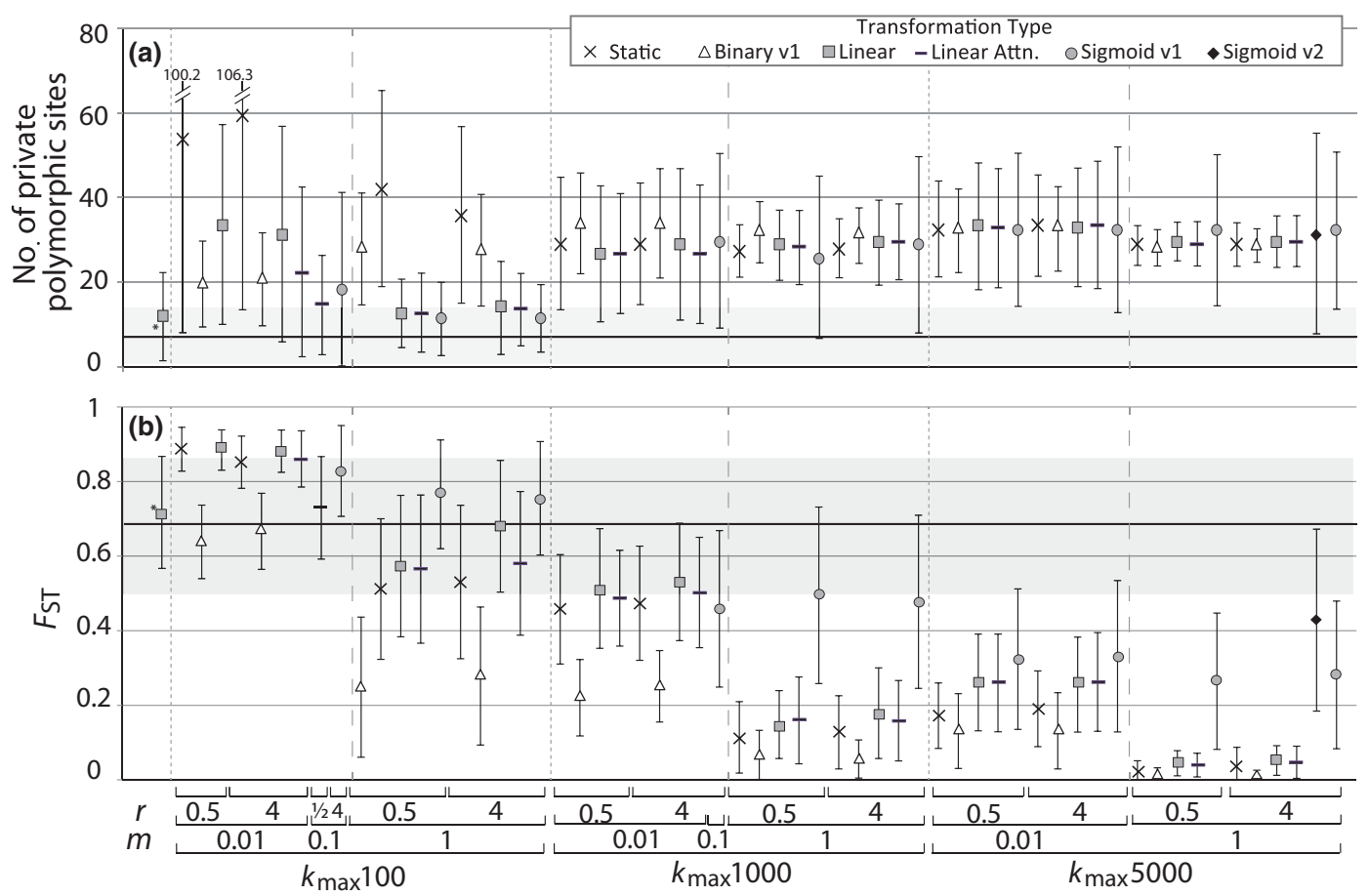

Fig. 6. Comparison of the impact of different demographic models on patterns of genetic variation, as characterized by: (A) the mean number of private polymorphic sites (PPS) and (B) Mean $F_{\mathrm{ST}}$. Black line depicts observed mean values for PPS and $F_{\mathrm{ST}}(7.25$ and 0.682 , respectively) and shaded area depicts the observed standard deviation for PPS and $F_{\mathrm{ST}}( \pm 6.69$ and 0.183 , respectively), and errors bars represent the standard deviation of values among replicate genetic simulations. ${ }^{*} K_{\max }=50, m=1, r=4$.

regarding the genetic consequences of distributional shifts hereafter refer to only those transformations for which all 12 focal populations were colonized under most demographic parameters (i.e. the results from the Static, Binary v1, Linear, Linear Attn. and Sigmoid v1 transformation; Table 1).

\section{Signatures of spatiotemporal variation and habitat heterogeneity}

As observed in other studies, increases in spatiotemporal variation and habitat heterogeneity had predictable effects on the genetic variation and both increased $F_{\mathrm{ST}}$ between demes (Wegmann et al. 2006; Knowles \& Serrano-Alvarado 2010). For example, there is a strong positive correlation between level of spatiotemporal variation and mean population $F_{\mathrm{ST}} \quad(r=0.749, n=12$, $P<0.001)$ when comparing among the three linear transformations (Static, Linear Attn. and Linear) at $K_{\max }=100$ and $m=1$. The primary difference among each is the level of spatiotemporal variation across transformations (Table 3, see also Fig. 5 for a graphic representation of how population sizes vary across time with differing transformations). Likewise, habitat heterogeneity was correlated with genetic diversity. For example, at $K_{\max }=100$ and $m=1$, both measures of habitat heterogeneity displayed strong correlations to mean population $F_{\mathrm{ST}}$ (coefficient of variance: $r=0.806, n=20$, $P<0.001$; Morin's I: $r=-0.792, n=20, P<0.001$ ).

Unlike previous studies (Wegmann et al. 2006), we found clear differences between models that include$\mathrm{d} /$ excluded the spatiotemporal dynamic. This might, in part, reflect the parameters considered here (which were chosen based on the biology of pikas), and perhaps, if we had considered a different parameter space, we might have identified situations where the genetic signatures associated with a spatiotemporal dynamic would be indistinguishable from those generated only by spatial heterogeneity. However, it no doubt also reflects the very simple spatial scenarios (e.g. no spatial autocorrelation across landscape in habitat heterogeneity) considered in pervious theoretical treatments (Wegmann et al. 2006). In our simulations, models lacking spatiotemporal variation resulted in higher genetic diversity values and higher variation among replicate data sets simulated for a given scenario (as observed in the very high PPS-values in the Static transformation) particularly when $K_{\max }$ was low. These PPS-values were considerably higher than observed in the actual genetic data.

Disentangling the separate contributions of spatiotemporal variation and habitat heterogeneity, and their relative importance to the resulting genetic variation is 
difficult (Zellmer \& Knowles 2009; Marko \& Hart 2011). This, in part, is because spatiotemporal variation requires some level of habitat heterogeneity. In fact, spatiotemporal variation simply changes the degree of spatial heterogeneity over time. Nevertheless, the biological mechanisms associated with specific genetic consequences, such as reduced genetic variation within populations, differ depending on whether a species experiences spatial heterogeneity vs. spatiotemporal variation during distributional shifts, even if both result in similar genetic values (Wegmann et al. 2006; Excoffier et al. 2009). Therefore, distinguishing which factor is contributing to patterns of genetic variation is motivated by the biological processes involved. For example, increases in habitat heterogeneity results in increased population isolation, and a concentration of coalescent events locally, whereas increases in spatiotemporal variation results in an increased frequency of population contractions of demes over time. Both carry the signature of reduced genetic diversity, but in the former case it reflects low levels of population connectivity, whereas in the latter it reflects changes in population size over time. If one would try to mitigate the effects of these two processes (e.g. make conservation decisions to avoid local populations with reduced genetic diversity), different strategies would be more or less effective depending on the factor underlying the reduced genetic diversity. For example, ascribing patterns of variation entirely to spatial heterogeneity could suggest that specific habitat corridors might be key to reducing the isolation across populations, thereby avoiding reductions in genetic diversity. However, if indeed temporal heterogeneity was contributing to the reduced genetic diversity within local demes, management efforts might be more effective if they were aimed at minimizing the demographic fluctuations (such as increasing the size of a reserve) rather than focusing on the connections across space.

\section{Similarity between simulated data and empirical data}

With respect to the suite of conditions and demographic models considered here, several resulted in composite values ( $F_{\mathrm{ST}}$ and PPS, Fig. 6$)$ that are almost identical to the observed values. Conversely, few produced patterns of genetic variation (at least for the summary statistics used to characterize patterns of genetic variation, Fig. 6) that strongly matched what was observed in the empirical data set (Figs S4 and S5, Supporting information). This shows that even if a limited set of summary statistics considered here, some modelled scenarios can be considered highly unlikely. Some of the differences between simulated and observed patterns of genetic variation is expected to be a result of the complicated biogeography of the focal species in relation to our simplistic classification of temporal dynamic (Galbreath et al. 2009, 2010). The inability to perform demographic models at higher resolutions could also contribute to differences between simulated and observed patterns of genetic variation and in our study; this created a tradeoff between spatial resolution and geographic extent of the models. For example, both factors could result in reduced abilities of models to account for the actual distribution of all relevant ancestors (owing to spatial clipping) or an oversimplification of geospatial data (owing to coarse spatial resolution).

To examine whether the different models can be discerned under a simple history characterized by a pattern of IBD, we selected a subsample of pika populations in which the pattern of variation corresponded to IBD (i.e. Subset 1; see details in methods). In this data set, there was less variation among the simulated data and many simulations resulted in high correlation coefficients and were able to emulate patterns of genetic variation across populations similar to the observed genetic data (Figs S4 and S5, Supporting information). In Subset 2, a situation where there is little evidence of IBD, the degree of correspondence between simulated data and empirical data varied greatly among models, with several models resulting in strong correlations $(r>0.5$, Figs S4 and S5, Supporting information). These results demonstrate that some of the models were able to capture more complicated biogeographic scenarios and are not limited to simple situations where IBDs is expected (a situation where differences among models were less pronounced). In some cases results were surprising. For example, the static model consistently produced results which were more similar to observed genetic patterns than many more complicated environmental models. One explanation may be due to the path of initial colonization of the models. In the static demographic models, the environmental variables $\left(K_{l}\right.$ and $\left.F_{l}\right)$ are the same throughout the simulation; however, initial populations colonize the landscape from a shared LGM distribution. In the Subset 2 data sets, one of the most apparent differences between the models was the manner under which the Raft Mountain population was colonized (see Fig. 1 for map). In most simulations, the common ancestor dispersed across the Rocky Mountains from a recent common ancestor to McBride and Grande Cache; however, in the static simulations, the common ancestor to Raft Mountain radiated quickly counterclockwise around the Rocky Mountains, a pattern more congruent with the observed genetic data (see Appendix S2, Supporting information). Other demographic models might better emulate this history if spatial resolution was increased (a current limitation of these models) or 


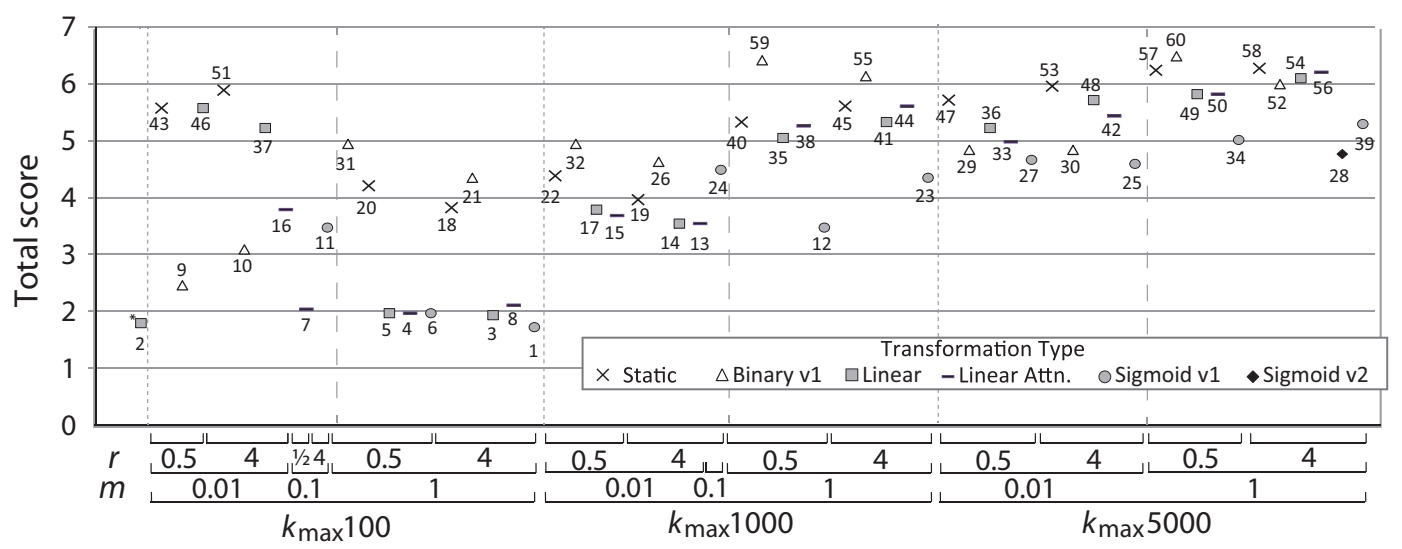

Fig. 7. Differences in the similarity between genetic data simulated under different models and the empirical data. Differences were evaluated by the total transformation correspondence score that was calculated as the sum of relative values of how similar each summary statistic is to observed genetic data. A model with identical summary statists to the observed data would have a score of zero. Each model is labelled with its corresponding rank (shown in parentheses). ${ }^{*} K_{\max }=50, m=1, r=4$.

Table 2 Comparisons showing that transformations significantly impacted patterns of genetic variation, as evaluated by composite summary statistics calculated for the simulated data

\begin{tabular}{|c|c|c|c|c|c|}
\hline$F_{\mathrm{ST}} / \mathrm{PPS}$ & Static & Binary v1 & Linear & $\begin{array}{l}\text { Linear } \\
\text { Attn. }\end{array}$ & Sigmoid v1 \\
\hline Static & & 0.293 & 0.006 & 0.007 & 0.003 \\
\hline Binary v1 & 0.008 & & 0.051 & 0.067 & 0.019 \\
\hline Linear & 0.352 & 0.002 & & 1 & 1 \\
\hline Linear Attn. & 1 & 0.004 & 1 & & 1 \\
\hline Sigmoid v1 & 0.011 & $<0.001$ & 0.119 & 0.031 & \\
\hline
\end{tabular}

Probability values from post-hoc pairwise comparisons of Mean $F_{\mathrm{ST}}$ (lower-left matrix) and the number of private polymorphic sites (PPS, upper-right matrix) among transformations (data simulated with $K_{\max }=100, m=1$ ). These comparisons identified significant differences $(P<0.05)$ in summary statistic values between corresponding transformations.

increased spatial extent allowing for the option of colonization from more western ancestors.

The top 8 ranked models were among three different transformations (linear, linear attenuated, sigmoid; Fig. 7, Table 1). Of those, seven had a $K_{\max }=100 \mathrm{a}$ $m=1$. Under these parameters, the static and binary models ranked the lowest (Fig. 7); producing summary statistic values that differed considerably from the observed genetic values. Comparisons among transformations revealed that four of the five transformations produced statistically different composite summary statistics (Table 2), reaffirming that different transformations are important parameters to consider when using SDMs in demographic models. In pikas, these results support that models based on highly heterogeneous landscapes that exhibit continuous spatial temporal variation (i.e. Linear, Linear Attn. and Sigmoid v1) significantly increased model's ability to emulate the empirical data set (Mann-Whitney $U$ comparison among $K_{\max } 100$ transformation rank scores: $U=27, n_{1}=15 n_{2}=12, P<0.001$; for data see Tables 1 and $\mathrm{S1}$, Supporting information).

\section{Implications for understanding the genetic consequences of climate-induced distributional shifts in pikas}

Even though our exploration is focused on the challenges and promise of the proposed integrative approach, as opposed to providing a precise estimate of the biogeographic and demographic history of the pikas (which is beyond the scope of this study, in part, because of limitations associated with the sufficiency of available summary statistics to characterize the genetic data for complex models; see Beaumont et al. 2010), the results nevertheless have some interesting implications. Based on consideration of those scenarios that produced results similar to those observed in the empirical data $\left(\right.$ mean $F_{\mathrm{ST}}$ of observed data $=0.682 \pm 0.183($ mean $\pm \mathrm{SD})$ vs. $0.717,0.669 \pm 0.191,0.3091 \pm 0.181$, and $0.1738 \pm 0.132$ for carrying capacities with $K_{\max }=50,100,1000$ and 5000 , respectively), the simulations suggest since the LGM, the carrying capacity was extremely low for pikas with a maximum number effective individuals of 50100 per $12 \mathrm{~km}^{2}$. Our results also suggest that pikas, at least occasionally, dispersed more than $6 \mathrm{~km}$ a year, given that seven of the top 8 ranked models possessed a high migration rate ( $12 \mathrm{~km}$ per generation).

The relative changes in population sizes in our linear and sigmoid demographic simulations (Fig. 5) mirror recent results from Bayesian skyline analyses of 
Table 3 Indices of habitat and temporal heterogeneity used to simulate the genetic data

\begin{tabular}{|c|c|c|c|}
\hline & \multirow[b]{2}{*}{$\begin{array}{l}\text { Spatiotemporal } \\
\text { Variation } \\
\text { Index (SVI) }\end{array}$} & \multicolumn{2}{|c|}{ Habitat heterogeneity } \\
\hline & & $\begin{array}{l}\text { Coefficient of } \\
\text { variation }\end{array}$ & Morin's $I$ \\
\hline Static & 0.000 & 1.599 & 0.653 \\
\hline Binary v1 & 0.586 & 0.859 & 0.797 \\
\hline Linear & 0.258 & 1.157 & 0.732 \\
\hline Linear Attn. & 0.151 & 1.358 & 0.726 \\
\hline Sigmoid v1 & 0.260 & 2.559 & 0.625 \\
\hline
\end{tabular}

Spatiotemporal variation: Low Spatiotemporal Variation Index (SVI) values reflect scenarios with little spatiotemporal variation. A temporal variation index value of 1 would depict that each cell in the simulation changed from the maximum to minimum carrying capacity value (or vice versa) during the simulations. Habitat heterogeneity: To quantify landscape heterogeneity, we evaluated two different aspects of heterogeneity-overall variation and the magnitude of spatial autocorrelation. Higher coefficients of variation represent higher levels habitat variation. Morin's I is measure of global spatial autocorrelation. In general, a Moran's Index value near +1.0 indicate strong clustering of habitat values while an index value near -1.0 indicate highly dispersed habitat values and values near zero indicate a random spatial distribution of habitat values.

molecular data (Galbreath et al. 2009), displaying a recent population reduction since the LGM. Interestingly the population sizes in the demographics were based entirely on $K_{l}$, supporting, at least in this case, the use of SDMs are suitable for calculating carrying capacity landscapes. Our demographic models (based on explicit climate, life history and geography data) provide independent support towards other recent results inferred solely from genetic data. For example, recent researchers demonstrated that patterns of pika differentiation appeared to be determined by an interaction between gene flow caused by glacial expansion and extinctions caused by interglacial retraction (Galbreath et al. 2009). Further, the authors argued that contemporary isolation is not a good predictor of either lineage diversity or historical barriers and during glacial periods, gene flow should occur among sky island populations and result in lineage cohesion within contiguous mountains. Lastly, they observed that all pika lineages exhibit a genetic signature of recent population decline; attributing it to a retraction during the Holocene (Galbreath et al. 2010). Each of these factors corroborate with our demographic simulations that produced genetic data similar to the empirical data set. This suggests that these methods can contribute to a more general understand of the genetic effects of climate-driven range fluctuations in other alpine organisms while accounting for species-specific life histories.

\section{Conclusions}

The information contained in SDMs has the potential to be used to generate species-specific predictions about patterns of genetic variation. Our results show how habitat heterogeneity and life history characteristics (e.g. differences in local carrying capacities and migration potentials) have a significant impact on the distribution of genetic variation among populations, as well as local population diversities. One important implication of this work is that the genetic consequences of distributional shifts will differ among taxa, even if they are moving across the same landscape. The application of these methods has considerable potential for other fields (i.e. landscape genetics and conservation biology) that focus on shorter temporal scales and smaller spatial scales. For example, these methods can be used to model the genetic consequences of changes of land use or the genetic consequences of climate change on conserved areas.

Because our results also demonstrate that the transformation used to convert information from SDMs into a demographic model has a distinct genetic signature, it is important to consider alternative transformations. We discuss how the transformation could itself be a parameter that is estimated with model-based inference (e.g. using an $A B C$ approach), or alternatively, considered sequentially to explore the robustness of a study's conclusions to different transformations. We also show that in contrast to previous theoretical treatments (e.g. Wegmann et al. 2006), the spatiotemporal dynamic can have a significant impact on patterns of genetic variation that differ from the genetic consequences associated with spatial heterogeneity, particularly when carrying capacities are reduced.

Comparing the results from simulated genetic data for different demographic scenarios to patterns of genetic variation observed in empirical data for the American Pika, O. princeps (Galbreath et al. 2009), the lack of similarity under most demographic scenarios means such conditions are unlikely to characterize the biogeographic and demographic history. Interestingly, the general concordance between some simulated scenarios and the empirical data involving changes in population size over time (as predicted from the SDMs and tested with the genetic data) corroborate suggestions from recent (and independent) results from Bayesian skyline analyses of molecular data (Galbreath et al. 2009). However, the development of additional summary statistics would be helpful for corroborating such aspects of the demographic history (i.e. confirming that alternative demographic scenarios would not produce similar genetic signatures) (Knowles \& Maddison 2002; Knowles 2009; Beaumont et al. 2010). Validation of the 
spatiotemporal demographic models is also important and needs to be considered, particularly if this method is used to test statistical phylogeographic hypotheses. Likewise, the development of additional summary statistics will no doubt be key in statistical evaluation of complex models like the ones proposed here, and is an area that needs attention for the potential of $A B C$ approaches to be fully realized.

\section{Acknowledgements}

We are grateful to Mathias Currat for making several changes to the SPLATCHe 2 program. We are indebted to Kurt Galbreath for considerable cooperation and support throughout this project, to Lauren Chan, Diego Alvarado-Serrano and Qixin He for many discussions at several phases during this project. This material is based upon work suppported by the National Science Foundation under Grand No. 0905905.

\section{References}

Andersen KK, Azuma N, Barnola JM et al. (2004) Highresolution record of Northern Hemisphere climate extending into the last interglacial period. Nature, 431, 147-151.

Beaumont M, Zhang W, Balding D (2002) Approximate Bayesian computation in population genetics. Genetics, 162, 2025-2035.

Beaumont M, Rasmus N, Robert C et al. (2010) In defense of model-based inference in phylogeography. Molecular Ecology, 19, 436-446.

Braconnot P, Otto-Bliesner B, Harrison S et al. (2007) Results of PMIP2 coupled simulations of the Mid-Holocene and Last Glacial Maximum-Part 1: experiments and large-scale features. Climate of the Past, 3, 261-277.

Brant AC (1989) Mate choice and reproductive success of pikas. Animal Behaviour, 37, 118-132.

Brown JL, Knowles LL (2012) Data from: spatially explicit models of dynamic histories: examination of the genetic consequences of Pleistocene glaciation and recent climate change on the American Pika. Dryad Digital Repository DOI: 10.5061/dryad.6pq7jg8p.

Carnaval AC, Hickerson MJ, Haddad CFB, Rodrigues MT, Moritz C (2009) Stability predicts genetic diversity in the Brazilian Atlantic forest hotspot. Science, 323, 785-789.

Carstens BC, Richards CL (2007) Integrating coalescent and ecological niche modeling in comparative phylogeography. Evolution, 61, 1439-1454.

Chan L, Brown JL, Yoder AD (2011) Integrating statistical genetic and geospatial methods brings new power to phylogeography. Molecular Phylogenetics and Evolution, 59, 523-537.

Currat M, Ray N, Excoffier L (2004) SPLATCHE: a program to simulate genetic diversity taking into account environmental heterogeneity. Molecular Ecology Notes, 4, 139-142.

Drummond AJ, Rambaut A, Shapiro B, Pybus OG (2005) Bayesian coalescent inference of past population dynamics from molecular sequences. Molecular Biology and Evolution, 22, 1185-1192.
Dumont BL, Payseur BA (2008) Evolution of the genomic rate of recombination in mammals. Evolution, 62, 276-294.

Dyke AS, Moore A, Robertson L (2003) Deglaciation of North America. Geological Survey of Canada. Open File 1574, 2 sheets.

Elith J, Phillips SJ, Hastie T, Dudík M, Chee YE, Yates CJ (2011) A statistical explanation of MaxEnt for ecologists. Diversity and Distributions, 11, 43-57.

Excoffier L, Lischer HEL (2010) Arlequin suite ver 3.5: a new series of programs to perform population genetics analyses under Linux and Windows. Molecular Ecology Resources, 10, 564-567.

Excoffier L, Novembre J, Schneider S (2000) SIMCOAL: a general coalescent program for the simulation of molecular data in interconnected populations with arbitrary demography. Journal of Heredity, 91, 506-510.

Excoffier L, Foll M, Petit RJ (2009) Genetic consequences of range expansions. Annual Review of Ecology Evolution and Systematics, 40, 481-501.

Galbreath KE, Hafner DJ, Zamudio K (2009) When cold is better: climate-driven elevation shifts yield complex patterns of diversification and demography in an alpine specialist (American Pika, Ochotona princeps). Evolution, 63, 2848-2863.

Galbreath KE, Hafner DJ, Zamudio KR, Agnew K (2010) Isolation and introgression in the Intermountain West: contrasting gene genealogies reveal the complex biogeographic history of the American Pika (Ochotona princeps). Journal of Biogeography, 37, 344-362.

Gelman A, Meng XL (1996) Model checking and model improvement. In: Markov Chain Monte Carlo in Practice (eds Gilks WR, Richardson S and Spiegelhalter DJ), pp. 163-187. Chapman \& Hall, London.

Graham CH, van der Wal J, Phillips SJ, Moritz C, Williams SE (2010) Dynamic refugia and species persistence: tracking spatial shifts in habitat through time. Ecography, 33, 10621069.

Grayson DK (2005) A brief history of Great Basin pikas. Journal of Biogeography, 32, 2103-2111.

Hafner DJ (1993) North American Pika (Ochotona princeps) as a Late Quaternary biogeographic indicator species. Quaternary Research, 39, 373-380.

Hafner DJ, Sullivan RM (1995) Historical and ecological biogeography of Nearctic pikas (Lagomorpha, Ochotonidae). Journal of Mammalogy, 76, 302-321.

Hewitt GM (1996) Some genetic consequences of ice ages, and their role in divergence and speciation. Biological Journal of Linnaean Society, 58, 247-276.

Hijmans RJ, Cameron SE, Parra JL, Jones PG, Jarvis A (2005) Very high resolution interpolated climate surfaces for global land areas. International Journal of Climatology, 25, 1965-1978.

Hoffman RS, Smith AT (2005) Order Lagomorpha. In: Mammal Species of the World: A Taxonomic and Geographic Reference, 3rd edn (eds Wilson DE and Reeder DM), pp. 185-211. Johns Hopkins University Press, Baltimore, Maryland, 2 vols. ISBN 978-0-8018-8221-0.

Jensen-Seaman MI, Furey T, Payseur B et al. (2004) Comparative recombination rates in the rat, mouse, and human genomes. Genome Research, 14, 528-538.

Knowles LL (2009) Statistical phylogeography. Annual Review of Ecology and Systematics, 40, 593-612.

Knowles LL, Alvarado-Serrano DF (2010) Exploring the population genetic consequences of the colonization process 
with spatio-temporally explicit models: insights from coupled ecological, demographic and genetic models in montane grasshoppers. Molecular Ecology, 19, 3727-3745.

Knowles LL, Maddison WP (2002) Statistical phylogeography. Molecular Ecology, 11, 2623-2635.

Knowles LL, Richards CL (2005) Importance of genetic drifts during Pleistocene divergence as revealed by analysis of genomic variation. Molecular Ecology, 14, 4023-4032.

Knowles LL, Carstens BF, Keat ML (2007) Coupled genetic and ecological-Niche models to examine how past population distributions contribute to divergence. Current Biology, 17, 1-7.

Kumar S, Subramanian S (2002) Mutation rates in mammalian genomes. Proceedings of the National Academy of Sciences, USA, 99, 803-808.

Lohse K, James N, Stone GN (2011) Inferring the colonization of a mountain range-refugia vs. nunatak survival in high alpine ground beetles. Molecular Ecology, 20 (2): 394-408.

Leuenberger C, Wegmann D (2010) Bayesian computation and model selection without likelihoods. Genetics, 184, 243-252.

Maddison DR, Maddison WP (2005) MacClade 4: analysis of phylogeny and character evolution. Version 4.08a. http:// macclade.org.

Manolopoulou I, Legarreta L, Emerson BC, Brooks S, Tavaré S (2011) A Bayesian approach to phylogeographic clustering. Interface Focus, 1, 909-921.

Marko PB, Hart MW (2011) The complex analytical landscape of gene flow inference. Trends in Ecology and Evolution, 26, 448-456.

Mead JI (1987) Quaternary records of pika, Ochotona, in North America. Boreas, 16, 165-171.

Millar JS (1974) Success of reproduction in pikas, Ochotona princeps (Richardson). Journal of Mammalogy, 55, 527-542.

Morgan K, O'Loughlin S, Chen B et al. (2011) Comparative phylogeography reveals a shared impact of Pleistocene environmental change in shaping genetic diversity within nine Anopheles mosquito species across the Indo-Burma biodiversity hotspot. Molecular Ecology, 20, 4533-4549.

Neuenschwander S, Largiader CR, Ray N, Currat M, Vonlanthen P, Excoffier L (2008) Colonization history of the Swiss Rhine basin by the bullhead (Cottus gobio): inference under a Bayesian spatially explicit framework. Molecular Ecology, 17, 757-772.

Peacock MM (1997) Determining natal dispersal patterns in a population of North American Pikas (Ochotona princeps) using direct mark-resight and indirect genetic methods. Behavioral Ecology, 8, 340-350.

Peacock MM, Smith AT (1997) The effect of habitat fragmentation on dispersal patterns, mating behavior, and genetic variation in a pika (Ochotona princeps) metapopulation. Oecologia, 112, 524-533.

Phillips SJ, Anderson RP, Schapire RE (2006) Maximum entropy modeling of species geographic distributions. Ecological Modeling, 190, 231-259.

Ray N, Currat M, Berthier P, Excoffier L (2005) Recovering the geographic origin of early modern humans by realistic and spatially explicit simulations. Genome Research, 15, 1161-1167.

Ray N, Currat M, Foll M, Excoffier L (2010) SPLATCHE2: a spatially explicit simulation framework for complex demography, genetic admixture and recombination. Bioinformatics, 26, 2993-2994.
Richards CL, Carstens BC, Knowles LL (2007) Distribution modelling and statistical phylogeography: an integrative framework for generating and testing alternative biogeographical hypotheses. Journal of Biogeography, 34, 1833-1845.

Sims GE, Jun SR, Wu GA, Kim SH (2009) Whole-genome phylogeny of mammals: evolutionary information in genic and nongenic regions. Proceedings of the National Academy of Sciences, USA, 106, 17077-17082.

Smith AT, Hanski I (1998) Long-term dynamics in a metapopulation of the American Pika. American Naturalist, 152, 530-542.

Smith AT, Ivins BL (1983) Colonization in a pika population: dispersal vs. philopatry. Behavioral Ecology Sociobiology, 13, 37-47.

Smith AT, Weston ML (1990) Ochotona princeps. Mammal Species, 352, 1-8.

Southwick CH, Golian SC, Whitworth MR, Halfpenny JC, Brown R (1986) Population density and fluctuations of Pikas (Ochotona princeps) in Colorado. Journal of Mammalogy, 67, 149-153.

Tapper SC (1973) The Spatial Organisation of Pikas (Ochotona), and Its Effect on Population Recruitment. Unpublished Ph.D. dissertation, University of Alberta, Edmonton, Alberta.

Veizer J, Godderis Y, Francois LM (2000) Evidence for decoupling of atmospheric $\mathrm{CO} 2$ and global climate during the Phanerozoic eon. Nature, 408, 698-701.

Verdu P, Austerlitz F, Estoup A et al. (2009) Origins and genetic diversity of pygmy hunter-gatherers from Western Central Africa. Current Biology, 19, 312-318.

Voje KL, Hemp C, Flagstad Ø, Saetre GP, Stenseth NC (2009) Climatic change as an engine for speciation in flightless Orthoptera species inhabiting African mountains. Molecular Ecology, 18, 93-108.

Wegmann D, Currat M, Excoffier L (2006) Molecular diversity after a range expansion in heterogeneous environments. Genetics, 174, 2009-2020.

Wiens JA, Stralberg D, Jongsomjit D, Howell CA, Snyder MA (2009) Niches, models, and climate change: assessing the assumptions and uncertainties. Proceedings of the National Academy of Sciences, USA, 106, 19729-19736.

Zellmer AJ, Knowles LL (2009) Disentangling the effects of historic versus contemporary landscape structure on population genetic divergence. Molecular Ecology, 18, 3593-3602.

J.L.B is a Postdoctoral Fellow whose research focuses on: 1. the interplay between spatial, genetic and evolutionary processes and 2. the behavioral ecology, evolution and taxonomy of poison frogs (family Dendrobatidae). His research addresses methodological and statistical issues involved in studying species diversification, species distribution patterns and community composition with an emphasis on geospatial analyses. L.LK is an Associate Professor whose research focuses on the demographic and selective processes driving species divergence. Her research also addresses the methodological and statistical issues involved in studying species diversification, phylogeography, and phylogenetic relationships of recently diverged and rapidly speciating taxa. 


\section{Data accessibility}

Genbank accessions: cyt b/D-loop, EU568283-EU568340; MGF, EU591095-EU591291; PRKCI, EU591292-EU591463. PopSet accessions: cyt b/D-loop, 189490845; MGF, 189547216; PRKCI, 189547413. Population locations, Maxent locality data, GIS data sources, LGM fossil localities: Brown et al. (2012) DRYAD entry doi: 10.5061/dryad.6pq7jg8p.

\section{Supporting information}

Additional supporting information may be found in the online version of this article.

Appendix S1 The following populations were included in the simulations: name/locality.

Appendix S2 Effects of different transformations on demographic models $\left(K_{\max }=1000, r=0.01, m=0.1\right)$.

Appendix S3 Effects of different carrying capacities, growth and migration rates on demographic models based a linear transformed SDM.
Table S1 Additional simulations at $K_{\max }=100, m=1$.

Table S2 Pearson correlation $(r)$ between friction and carrying capacity landscapes.

Fig. S1 Known current distribution of the American Pika (Ochotona princeps).

Fig. S2 Carrying capacity landscapes transformations.

Fig. S3 Overview of creating temporally continuous landscapes for spatially explicit demographic simulation.

Fig. S4 Patterns of genetic divergence: Pairwise comparision of mean population $F_{\mathrm{ST}}$.

Fig. S5 Patterns of genetic divergence: PCA.

Please note: Wiley-Blackwell is not responsible for the content or functionality of any supporting information supplied by the authors. Any queries (other than missing material) should be directed to the corresponding author for the article. 